\title{
Combination of Xylan Depolymerizing and Debranching En- zymes Improves Digestion, Growth Performance and Intestinal Volatile Fatty Acid Profile of Piglets
}

\author{
Weiwei Wang1, Dawen Zheng', Zhenzhen Zhang', Qingyun Cao ${ }^{1}$, Hui Ye ${ }^{1}$, Changming Zhang1, Zemin Dong1, \\ Dingyuan Feng ${ }^{1, *}$ and Jianjun $\mathrm{Zuo}^{1, *}$ \\ ${ }^{1}$ College of Animal Science, South China Agricultural University, Guangzhou 510642, China \\ 2 AsiaPac Bio-Technology Co., Ltd, Dongguan 523808, China \\ * Correspondences: fengdy@scau.edu.cn (D.Y.F.), Tel.: +86-020-85280740; zuoj@scau.edu.cn (J.J.Z.), Tel.: \\ $+86-020-85283756$
}

Simple Summary: Many plant feedstuffs (e.g. cereal brans) are abundant in arabinoxylan (Abx) that serves as a typical anti-nutritional factor for monogastric animals. Abx comprises a backbone of $\beta$-D-(1-4)-xylopyranosyl units onto which L-arabinose and ferulic acid groups are linked as the side chains. Due to the complex structure, the efficient degradation of Abx may demand an enzyme consortium consisting of depolymerizing enzymes such as endo-xylanase (Xyn) and debranching enzymes such as arabinofuranosidase (Afd) and feruloyl esterase (FE). To data, information about the potential synergy among Xyn, Afd and FE on degradation of Abx in plant ingredients along with the application of combination of these enzymes in pig diets is scarce. In this study, we found that combination of $\mathrm{Xyn}$, Afd and FE displayed a superiority over $\mathrm{Xyn}$ alone and its combination with Afd or FE in promoting degradation of Abx in multiple brans. Treatment with combination of Xyn, Afd and FE had advantages over Xyn alone in improving nutrient digestion, growth performance and intestinal volatile fatty acid profile of postweaning piglets fed bran-added diet. These indicated that combination of $\mathrm{Xyn}$, Afd and FE could be used as a prominent enzyme consortium for improving growth and gut health of piglets.

\begin{abstract}
This study was aimed to investigate the effect of xylan depolymerizing enzyme namely endo-xylanase (Xyn) combined with debranching enzymes namely arabinofuranosidase (Afd) and feruloyl esterase (FE) on digestion, growth performance and intestinal volatile fatty acid profile of piglets. The in vitro experiments were firstly conducted to examine the enzymological properties of Xyn, Afd and FE, the synergy among these enzymes, together with the effect of combination of these enzymes on digestion of piglet diet. The in vivo experiment was then implemented by allocating 27035 -d-old postweaning piglets into 3 treatment groups: control group, Xyn group and $(\mathrm{Xyn}+\mathrm{Afd}+\mathrm{FE})$ group. Each group had 6 replicates (15 piglets/replicate). The results revealed a satisfying thermostability and $\mathrm{pH}$ stability of Xyn, Afd and FE. Combination of Xyn, Afd and FE had a superiority $(P<0.05)$ over Xyn alone and its combination with Afd or FE in promoting degradation of different bran fibers rich in arabinoxylan (Abx). Treatment with combination of Xyn, Afd and FE had advantages over Xyn alone to induce increasing trends $(P<0.10)$ of in vitro digestibility of dietary nutrients (dry matter, crude protein, crude ash and gross energy) and piglet growth performance (average daily gain, final body weight and feed efficiency), concurrent with a reduction $(P<$ $0.05)$ of diarrhea rate and increases $(P<0.05)$ in cecal acetic acid, butyric acid and total volatile fatty acids concentrations as well as $\mathrm{pH}$ value of piglets. Collectively, combination of $\mathrm{Xyn}$, Afd and FE was efficient in benefiting degradation of Abx in brans, as well as improving digestion, growth performance and intestinal volatile fatty acid profile of piglets.
\end{abstract}

Keywords: depolymerizing enzyme; debranching enzyme; xylanase; arabinofuranosidase; feruloyl esterase; arabinoxylan; digestion; growth performance; volatile fatty acid; piglet 


\section{Introduction}

Arabinoxylan (Abx) exemplifies the typical non-starch polysaccharide in plentiful plant-derived feedstuffs such as cereal grains and brans [1], comprising a linear backbone of $\beta$-D-(1-4)-xylopyranosyl units onto which L-arabinose units are linked as the major side chains [2]. Generally, arabinose residues that attach to the C-2 and/or C-3 position of Abx backbone can be further substituted in the O-5 position by ferulic acid ester groups $[2,3]$. Abx is resistant to digestion of endogenous enzymes of animals, which causes viscous digesta with subsequent intestinal disorders such as reduced digestibility, increased pathogen load, gut leakage and inflammation, thus being characterized as a critical anti-nutritional factor for monogastric animals especially for those at young age [4,5]. These raise a necessity to explore strategies to degrade dietary Abx that may benefit intestinal digestion and growth performance of animals.

Enzymatic treatment has been evidenced as a promising approach to decompose polysaccharides in crop products [6,7]. Because of the complex molecular structure, the efficient degradation of Abx in diet may demand an enzyme consortium consisting of depolymerizing enzymes such as endo-xylanase (Xyn) and debranching enzymes such as arabinofuranosidase (Afd) and feruloyl esterase (FE) $[3,8]$. Thereinto, Xyn stochastically cleaves the $\beta$-1,4-glycosidic bonds within Abx backbone and produces smaller polysaccharides or oligosaccharides [3], which can be used as prebiotics to improve intestinal health by targeting host gut microbiota [4,9]. However, the presence of side chains fortifies the degradation resistance of Abx backbone through impeding recognition of cleavage sites in the backbone by Xyn $[8,10]$. This effect may be overwhelmed by the action of debranching enzymes, among which Afd removes the arabinose residues to expose more cleavage sites and provide a convenience for Xyn action [3,10], while FE breaks ferulic acid ester bonds cross-linked to arabinose residues to liberate ferulic acid [11,12]. These actions aid in simplifying the molecular structure of Abx and enhancing Xyn accessibility to produce reducing sugars [13]. Accordingly, there can be an efficient degradation of Abx in plant-sourced ingredients under the synergy among Xyn, Afd and FE, which may in turn diminish the viscosity of intestinal content, favoring a sufficient contact between digesta and digestive enzymes with a resultant benefit on digestion of dietary nutrients [4,5]. More importantly, the efficient degradation of Abx and the cleavage of ferulic acid ester bonds linking feruloyl residues of Abx with other cell wall components under the synergy among Xyn, Afd and FE probably prompt cell wall fragmentation of plant ingredients, which in turn profits the access of digestive enzymes to cell wall or intracellular components [14-16], presumptively triggering an enhanced digestion of plant ingredients with a subsequent promotion of growth and health of animals fed plant feedstuff-based diet. Although there were numerous studies confirming the positive effects of Xyn in combination with Afd on chicken growth performance and health condition [17-19], information about the synergistic action among Xyn, Afd and FE on the improvements of growth and gut health of piglets is scarce.

Comprehensively, we raised the assumption that the synergy among Xyn, Afd and FE was more favorable to result in an enhanced degradation of Abx with a destruction of cell wall of certain plant feedstuffs, possibly conducing to ameliorate the feeding effect of them. To test this hypothesis, the current study was conducted to investigate the effects of combination of Xyn, Afd and FE on degradation of cereal brans rich in Abx as well as growth and health of postweaning piglets received bran-added diet.

\section{Materials and Methods}

\subsection{Materials}

Xylose and arabinose were purchased from Aladdin (Shanghai, China). Beechwood xylan, p-nitrophenyl- $\alpha$-L-arabinofuranoside, $\alpha$-amylase, protease, glucosidase, pepsin and cellulase were purchased from Sigma-Aldrich (Shanghai, China). Methyl ferulate was purchased from Alfa Aesar (Beijing, China). Xyn (EC 3.2.1.8), Afd (EC 3.2.1.55) and FE (EC 3.1.1.73) were all provided by AsiaPac Co., Ltd (Dongguan, China). Trypsin and chymotrypsin were purchased from Amresco (Shanghai, China). Dialysis bag and pan- 
creatin were derived from Viskase (Beijing, China) and Lanxu Biotech. Co. (Hefei, China), respectively. Wheat bran and oat bran were provided by Xingye Biotech. Co. (Dongguan, China). Destarched wheat bran (DSWB) was prepared according to the method of Mukherjee et al. [20]. All other chemicals and solvents used in this study were of analytical grade.

\subsection{Enzymatic characteristics assay}

2.2.1. Determination of enzyme activities

The activity of Xyn was determined using beechwood xylan as the substrate [21]. The reaction mixture consisted of $200 \mu \mathrm{L}$ properly diluted enzyme and $1.8 \mathrm{~mL}$ of 10 $\mathrm{mg} / \mathrm{mL}$ xylan in $0.05 \mathrm{M}$ citric-Na2HPO4 (pH 5.0). After incubation at $40^{\circ} \mathrm{C}$ for $10 \mathrm{~min}$, the enzymatic activity was terminated by adding $2 \mathrm{~mL} 3,5$-dinitrosalicylic acid reagent (DNS) followed by boiling for $10 \mathrm{~min}$. This mixture was then cooled down for quantifying reducing sugar using DNS method with xylose as a standard [21].

Afd activity was determined by using p-nitrophenyl $\alpha$-L-arabinofuranoside as the substrate [22]. The reaction mixture consisted of $25 \mu \mathrm{L}$ properly diluted enzyme and 175 $\mu \mathrm{L}$ of $2 \mathrm{mM}$ substrate in $0.05 \mathrm{M}$ citric- $\mathrm{Na}_{2} \mathrm{HPO}_{4}$ ( $\mathrm{pH} 5.5$ ). After incubation at $40^{\circ} \mathrm{C}$ for 15 min, the enzymatic reaction was terminated by adding $200 \mu \mathrm{L}$ of $2 \mathrm{M} \mathrm{Na}_{2} \mathrm{CO}_{3}$. The concentration of p-nitrophenol was measured at optical density of $410 \mathrm{~nm}$.

For FE activity assay, methyl ferulate was used as the substrate [21]. The reaction mixture consisted of $10 \mu \mathrm{L}$ properly diluted enzyme and $190 \mu \mathrm{L}$ of $0.1 \mathrm{M}$ substrate in 0.05 $\mathrm{M}$ citric-Na2 $\mathrm{HPO}_{4}\left(\mathrm{pH}\right.$ 5.5). After incubation at $40^{\circ} \mathrm{C}$ for $15 \mathrm{~min}, 100 \mu \mathrm{L}$ acetonitrile was added into this mixture to terminate the enzymatic reaction. Ferulic acid was quantified by high performance liquid chromatography (Shimadzu SIL-20A, Japan) [21].

One unit of Xyn, Afd and FE was defined as the amount of enzyme required to generate $1 \mu \mathrm{mol}$ of product equivalent per min from the corresponding substrates under the standard assay conditions. Each reaction was performed in triplicate.

\subsubsection{Influence of $\mathrm{pH}$ and temperature on enzymes activities}

The influence of $\mathrm{pH}$ on enzyme activities was detected by measuring the relative activities of $\mathrm{Xyn}$, Afd and FE in $0.05 \mathrm{M}$ citric- $\mathrm{Na}_{2} \mathrm{HPO}_{4}$ buffer (pH 3.0 8.0) and glycine- $\mathrm{NaOH}$ ( $\mathrm{pH}$ 9.0 10.0) according to the method described above. Enzyme stability against $\mathrm{pH}$ was detected by determining the residual activities of these enzymes after incubation at $\mathrm{pH} 3.0$ for $30 \mathrm{~min}$.

The effect of temperature on enzyme activities was determined by measuring the relative activities of Xyn, Afd and FE at different temperatures $\left(30^{\circ} \mathrm{C} \sim 90^{\circ} \mathrm{C}\right)$ under the standard assay conditions described above. Thermostability stability of these enzymes was evaluated by determining the residual activities of these enzymes after incubation at $85^{\circ} \mathrm{C}$ for $3 \mathrm{~min}$.

\subsection{Effect of Xyn combined with Afd on degradation of Abx in DSWB}

Several tubes with $0.5 \mathrm{~g}$ DSWB each were divided into 6 groups (5 replicates/group): control, $\mathrm{X},(\mathrm{X}+\mathrm{A} 1),(\mathrm{X}+\mathrm{A} 2),(\mathrm{X}+\mathrm{A} 3)$ and $(\mathrm{X}+\mathrm{A} 4)$, which received the following treatments. Control: no enzymes; $X: 3 \mathrm{U} / \mathrm{g} X y n ;(X+A 1): 3 \mathrm{U} / \mathrm{g} X y n+0.001 \mathrm{U} / \mathrm{g}$ Afd; $(X+A 2): 3 \mathrm{U} / \mathrm{g}$ Xyn $+0.003 \mathrm{U} / g$ Afd; $(X+A 3): 3 \mathrm{U} / \mathrm{g}$ Xyn $+0.006 \mathrm{U} / \mathrm{g}$ Afd; $(X+\mathrm{A} 4): 3 \mathrm{U} / \mathrm{g}$ Xyn $+0.03 \mathrm{U} / \mathrm{g}$ Afd. Each treatment group was then added with phosphate buffer solution (PBS) to obtain 10 $\mathrm{mL}$ reaction system, followed by incubation in a constant temperature shaker $\left(40^{\circ} \mathrm{C}, 120\right.$ $\mathrm{rpm} / \mathrm{min}$ ) for $4 \mathrm{~h}$. After centrifugation at $3000 \mathrm{rpm} / \mathrm{min}$ for $3 \mathrm{~min}$, the supernatant was collected for quantifying the releasing of reducing sugar (RRS) using DNS method that represents the degradation of $\mathrm{Abx}$ [21].

\subsection{Effect of Xyn combined with FE on degradation of Abx in DSWB}

Several tubes with $0.5 \mathrm{~g}$ DSWB each were divided into 6 groups ( 5 replicates/group): control, $\mathrm{X},(\mathrm{X}+\mathrm{F} 1),(\mathrm{X}+\mathrm{F} 2),(\mathrm{X}+\mathrm{F} 3)$ and $(\mathrm{X}+\mathrm{F} 4)$, which received the following treatments. Control: no enzymes; $X: 3$ U/g Xyn; $(X+F 1): 3$ U/g Xyn + 0.001 U/g FE; $(X+F 2): 3$ U/g Xyn + 
0.003 U/g FE; (X+F3): 3 U/g Xyn + 0.006 U/g FE; (X+F4): 3 U/g Xyn + 0.03 U/g FE. Each treatment group was then added with PBS to obtain $10 \mathrm{~mL}$ reaction system and the supernatant was separated for quantification of the RRS using DNS method [21].

\subsection{Evaluation of synergy among Xyn, Afd and FE on degradation of Abx in different brans}

Wheat bran (WB) and oat bran (OB) were enzymolyzed as described previously [23]. The resulting zymolytes were used to extract the soluble fiber (SF) and insoluble fiber (IF) according to the report of Fahey et al. [24]. For evaluation of the synergy among Xyn, Afd and FE on the RRS from different Abx sources (WB-SF, WB-IF, OB-SF and OB-IF), several tubes with $0.5 \mathrm{~g}$ of each fiber were randomly allocated into 4 groups ( 5 replicates/group): $\mathrm{X},(\mathrm{X}+\mathrm{A}),(\mathrm{X}+\mathrm{F})$ and $(\mathrm{X}+\mathrm{A}+\mathrm{F})$, which received the following treatments. $\mathrm{X}: 3 \mathrm{U} / \mathrm{g} \mathrm{Xyn}$; $(X+A): 3$ U/g Xyn +0.001 U/g Afd; $(X+F): 3$ U/g Xyn +0.006 U/g FE; $(X+A+F): 3$ U/g Xyn + $0.001 \mathrm{U} / \mathrm{g}$ Afd $+0.006 \mathrm{U} / \mathrm{g}$ FE (Note: the dosages of Xyn, Afd and FE were selected according to the results in Figures 3 and 4). Each treatment group was then added with PBS to obtain $10 \mathrm{~mL}$ reaction system, the supernatant was separated for quantifying the RRS using DNS method [21].

The value of synergy degree between multiple enzymes was calculated as the ratio of the sum of the activities (generation amount of hydrolysate) of all enzymes to the activity of one of these enzymes [25]. If the ratio is less than 1.0, it reveals a negative synergy between enzymes, while the ratio exceeding 1.0 suggests a positive synergy between enzymes.

\subsection{Effect of combination of Xyn, Afd and FE on in vitro digestion of bran-added diet}

2.6.1. Preparation of in vitro digestion juice

Referring to previous studies in pigs [26,27], the gastric digestion juice was simulated by preparation of pepsin $(737.5 \mathrm{U} / \mathrm{mL}$ ) with HCL solution ( $\mathrm{pH} 2.0)$. The small intestinal digestion juice was simulated by preparing a mixture of amylase $(221.4 \mathrm{U} / \mathrm{mL})$, trypsin $(69.1 \mathrm{U} / \mathrm{mL})$ and chymotrypsin $(8.7 \mathrm{U} / \mathrm{mL})$ with deionized water. The large intestinal digestion juice was simulated by preparation of cellulase $(0.4 \mathrm{U} / \mathrm{mL})$ with deionized water.

\subsubsection{In vitro digestion of bran-added diet}

The composition and nutrient levels of bran-added diet based on the NRC requirement of swine [28] are shown in Table 1. The SDS-II simulated digestion system (Shenhua Biotech. Co. Ltd., China) was employed in simulating digestion of the above diet of piglets [26,27]. In brief, diet $(2 \mathrm{~g})$ was put into digestive tube sleeved with the MD34-14 dialysis bag, followed by digestion with $20 \mathrm{~mL}$ of simulated gastric juice together with one of three treatments (control, no enzymes; $\mathrm{X}, 3 \mathrm{U} / \mathrm{g} \mathrm{Xyn} ;(\mathrm{X}+\mathrm{A}+\mathrm{F}), 3 \mathrm{U} / \mathrm{g}$ Xyn $+0.001 \mathrm{U} / \mathrm{g} \mathrm{Afd}+0.006 \mathrm{U} / \mathrm{g} \mathrm{FE}$ ) at $39^{\circ} \mathrm{C}$ for $4 \mathrm{~h}$ in the digestion system. Immediately after simulated gastric digestion, the diet was treated by $2.2 \mathrm{~mL}$ of simulated small intestinal juice at $39^{\circ} \mathrm{C}$ for $16 \mathrm{~h}$ and simulated large intestinal juice at $39^{\circ} \mathrm{C}$ for $3.5 \mathrm{~h}$. Following these processes, the undegraded chyme was washed repeatedly with deionized water. Thereafter, the chyme residue was successively dried in an oven at $65^{\circ} \mathrm{C}$ and $105^{\circ} \mathrm{C}$ to constant weight, which was then cooled to measure the digestibility of nutrients (dry matter, crude protein, crude ash and gross energy) based on the method of Zhu et al. [27].

Table 1. The composition and nutrient levels of basal diet (as-fed basis)

\begin{tabular}{ll}
\hline \multicolumn{1}{c}{ IngredientsContent (\%)Corn60Soybean } & \multicolumn{1}{c}{ Content (\%)Corn60Soybean } \\
meal15Bran1.5Soybean oil0.45Puffed soybean6.9Fish & meal15Bran1.5Soybean oil0.45Puffed \\
meal4.5Whey powder7.75Dicalcium phosphate0.85Lime- & soybean6.9Fish meal4.5Whey pow- \\
stone1.1Salt0.3Lysine0.35Threonine0.15Methionine0.1Cho- & der7.75Dicalcium phos- \\
line0.2Silicon dioxide0.3Premix ${ }^{1}$ 0.55Total100Nutrient lev- & phate0.85Limestone1.1Salt0.3Ly- \\
\hline
\end{tabular}


elsDigestible energy (MJ/kg)14.43Metabolizable energy (MJ/kg)13.90Crude protein (\%)18.76Digestible lysine

sine0.35Threonine0.15Methio-

(\%)1.14Digestible methionine (\%)0.36Calcium nine 0.1 Choline 0.2 Silicon diox-

(\%)0.67Available phosphorus (\%)0.331 Supplied per kilo-

ide0.3Premix ${ }^{10.55 T o t a l 100 N u t r i e n t}$ gram of diet: $\mathrm{Cu}, 9.9 \mathrm{mg}$; (MJ/kg)14.43Metabolizable energy Zn, 142.5 mg; Fe, 242.5 mg; (MJ/kg)13.90Crude protein Mn, 68.7 mg; Se, 0.36 mg; I, (\%)18.76Digestible lysine (\%)1.14Di$0.64 \mathrm{mg}$; vitamin A, 11500 gestible methionine (\%)0.36Calcium IU; vitamin D3, $3250 \mathrm{IU} ;{ }^{(\%) 0.67 \text { Available phosphorus (\%)0.33 }}$ vitamin E, $91 \mathrm{mg}$; vitamin Sup$\mathrm{K} 3,3 \mathrm{mg}$; thiamin, $8.6 \mathrm{mg}$; riboflavin, $10.9 \mathrm{mg}$; pyridoxine, $9.3 \mathrm{mg}$; cobalamin, $0.2 \mathrm{mg}$; niacin, $62.6 \mathrm{mg}$; pantothenic acid, $26.8 \mathrm{mg}$; folic acid, $1.9 \mathrm{mg}$. 


\begin{tabular}{ll}
\hline & ne, \\
& 9.3 \\
& mg; \\
co- \\
bal- \\
amin, \\
o.2 \\
mg; \\
nia- \\
cin, \\
c2.6 \\
mg; \\
pan- \\
tothe \\
nic \\
acid, \\
26.8 \\
mg; \\
folic \\
acid, \\
1.9 \\
mg.
\end{tabular}

60Soybean

meal15Bran1.5Soybean oil0.45Puffed soybean6.9Fish meal4.5Whey powder7.75Dicalcium phosphate0.85Limestone1.1Salt0.3Lysine0.35Threonine0.15Methionine 0.1 Choline 0.2 Silicon dioxide0.3Premix ${ }^{1} 0.55$ Total100Nutrient levelsDigestible energy

Corn60Soybean meal15Bran1.5Soybean oil0.45Puffed soybean6.9Fish meal4.5Whey pow(MJ/kg)14.43Metabolizable energy (MJ/kg)13.90Crude protein der7.75Dicalcium phosphate0.85Limestone1.1Salt0.3Lysine0.35Threonine0.15Methionine0.1Choline0.2Silicon dioxide0.3Premix ${ }^{1} 0.55$ Total100Nutrient levelsDigestible energy (MJ/kg)14.43Metabolizable energy (MJ/kg)13.90Crude protein (\%)18.76Digestible lysine (\%)1.14Digestible methionine (\%)0.36Calcium (\%)0.67Available phosphorus (\%)0.33

Supplied per kilogram of (\%)18.76Digestible lysine (\%)1.14Digestible methionine $(\%) 0.36$ Calcium (\%)0.67Available phosphorus (\%) $0.33^{1}$

Supplied per diet: $\mathrm{Cu}, 9.9 \mathrm{mg} ; \mathrm{Zn}, 142.5$ kilo$\mathrm{mg}$; Fe, $242.5 \mathrm{mg}$; Mn, 68.7 gram $\mathrm{mg}$; Se, $0.36 \mathrm{mg}$; I, $0.64 \mathrm{mg}$; of vitamin A, $11500 \mathrm{IU}$; vitamin D3, 3250 IU; vitamin diet: E, $91 \mathrm{mg}$; vitamin $\mathrm{K} 3,3 \mathrm{mg}$; $\mathrm{Cu}$, thiamin, $8.6 \mathrm{mg}$; riboflavin, $10.9 \mathrm{mg}$; pyridoxine, 9.3 $\mathrm{mg}$; cobalamin, $0.2 \mathrm{mg}$; 9.9 $\mathrm{mg}$; $\mathrm{Zn}$, niacin, $62.6 \mathrm{mg}$; panto142.5 thenic acid, $26.8 \mathrm{mg}$; folic $\mathrm{mg}$; $\mathrm{Fe}$, acid, $1.9 \mathrm{mg}$. 


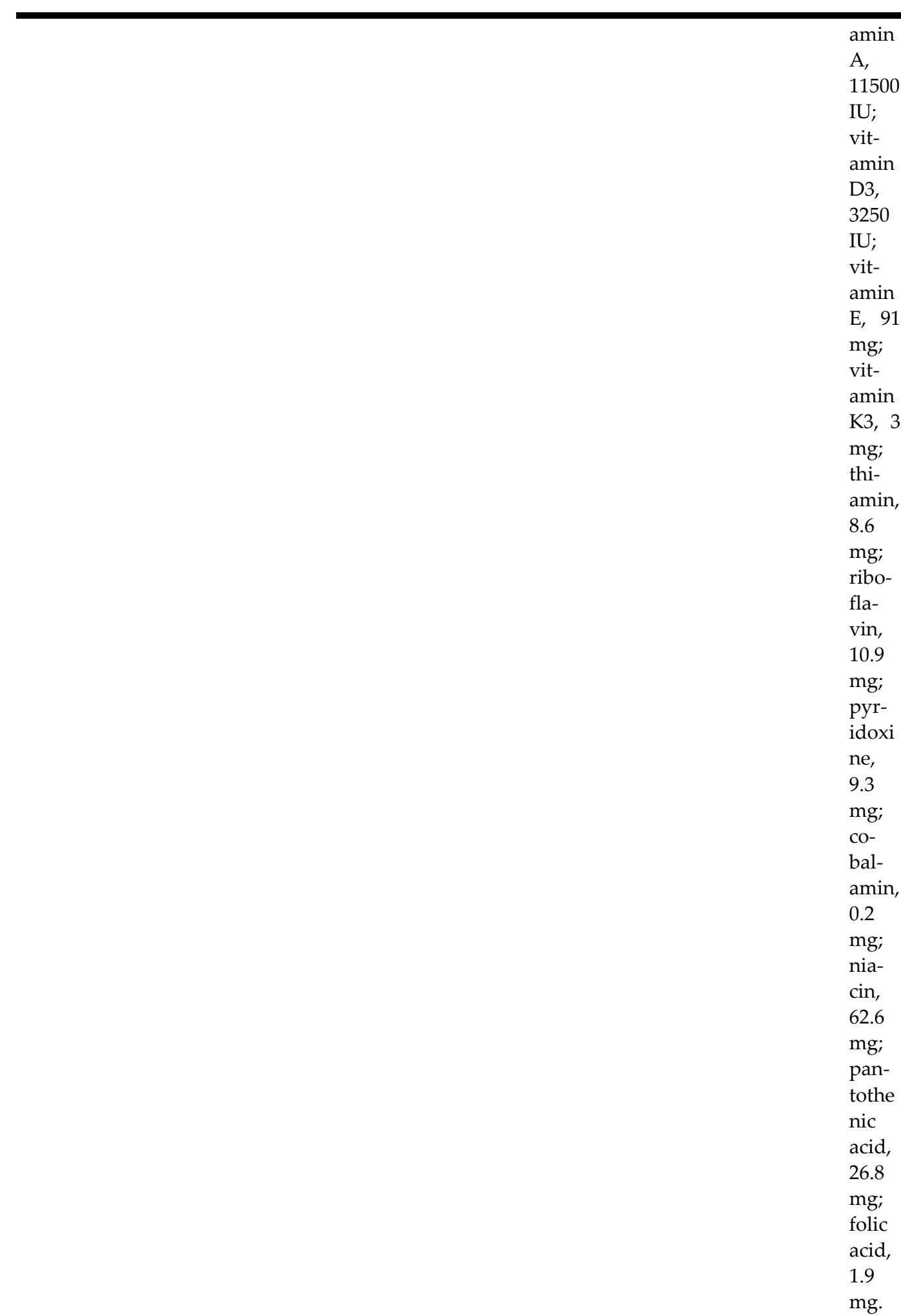

Soybean meal15Bran1.5Soybean oil0.45Puffed soybean6.9Fish meal4.5Whey powder7.75Dicalcium phosphate0.85Limestone1.1Salt0.3Lysine 0.35 Threonine0.15Methionine 0.1 Choline 0.2 Silicon dioxide0.3Premix ${ }^{1} 0.55$ Total100Nutrient levelsDigestible energy (MJ $/ \mathrm{kg}) 14.43$ Metabolizable energy (MJ/kg)13.90Crude protein (\%)18.76Digestible lysine (\%)1.14Digestible methionine (\%)0.36Calcium (\%)0.67Available phosphorus (\%) 0.33 
mg; Se, 0.36 mg; I, 0.64 mg; gestible methionine (\%)0.36Calcium vitamin A, $11500 \mathrm{IU}$; vita- (\%)0.67Available phosphorus (\%)0.331 min D3, $3250 \mathrm{IU}$; vitamin E, $91 \mathrm{mg}$; vitamin $\mathrm{K} 3,3 \mathrm{mg}$; thiamin, $8.6 \mathrm{mg}$; riboflavin, $10.9 \mathrm{mg}$; pyridoxine, 9.3 $\mathrm{mg}$; cobalamin, $0.2 \mathrm{mg}$; niacin, $62.6 \mathrm{mg}$; pantothenic acid, $26.8 \mathrm{mg}$; folic acid, $1.9 \mathrm{mg}$.

Sup-

plied

per

kilo-

gram

of

diet:

$\mathrm{Cu}$,

9.9

mg;

Zn,

142.5

mg;

$\mathrm{Fe}$,

242.5

mg;

$\mathrm{Mn}$,

68.7

$\mathrm{mg}$;

Se,

0.36

mg; I,

0.64

mg;

vit-

amin

A,

11500

IU;

vit-

amin

D3,

3250

IU;

vit-

amin

E, 91

mg;

vit-

amin

K3, 3

mg;

thi-

amin,

8.6

$\mathrm{mg}$;

ribo-

fla-

vin,

10.9

mg;

pyr-

idoxi

ne,

9.3

mg;

CO-

bal-

amin, 
Bran1.5Soybean oil0.45Puffed soybean6.9Fish meal4.5Whey powder7.75Dicalcium phosphate 0.85 Limestone1.1Salt0.3Lysine 0.35Threonine0.15Methionine 0.1 Choline $0.2 S$ ilicon dioxide 0.3 Premix ${ }^{10.55 T o-}$ tal100Nutrient levelsDigestible energy (MJ/kg)14.43Metabolizable energy (MJ/kg)13.90Crude protein (\%)18.76Digestible lysine (\%)1.14Digestible methionine (\%)0.36Calcium (\%)0.67Available phosphorus (\%)0.33 ${ }^{1}$ Supplied per kilogram of diet: $\mathrm{Cu}, 9.9 \mathrm{mg}$; $\mathrm{Zn}, 142.5 \mathrm{mg}$; Fe, $242.5 \mathrm{mg}$; $\mathrm{Mn}, 68.7 \mathrm{mg}$; Se, $0.36 \mathrm{mg}$; I, $0.64 \mathrm{mg}$; vitamin A, 11500

1.5Soybean oil0.45Puffed soybean6.9Fish meal4.5Whey powder7.75Dicalcium phosphate0.85Limestone1.1Salt0.3Lysine0.35Threonine0.15Methionine $0.1 C h o l i n e 0.2 S$ ilicon dioxide0.3Premix ${ }^{1} 0.55$ Total100Nutrient levelsDigestible energy (MJ/kg)14.43Metabolizable energy (MJ/kg)13.90Crude protein (\%)18.76Digestible lysine (\%)1.14Digestible methionine (\%)0.36Calcium (\%)0.67Available phosphorus (\%) $0.33^{1}$ IU; vitamin D3, 3250 IU; vitamin E, $91 \mathrm{mg}$; vitamin $\mathrm{mg}$; $\mathrm{K} 3,3 \mathrm{mg}$; thiamin, $8.6 \mathrm{mg}$; $\mathrm{Fe}$, riboflavin, $10.9 \mathrm{mg}$; pyri242.5 doxine, $9.3 \mathrm{mg}$; cobalamin, $0.2 \mathrm{mg}$; niacin, $62.6 \mathrm{mg}$; pantothenic acid, $26.8 \mathrm{mg}$; mg; $\mathrm{Mn}$, 68.7 folic acid, $1.9 \mathrm{mg}$. 


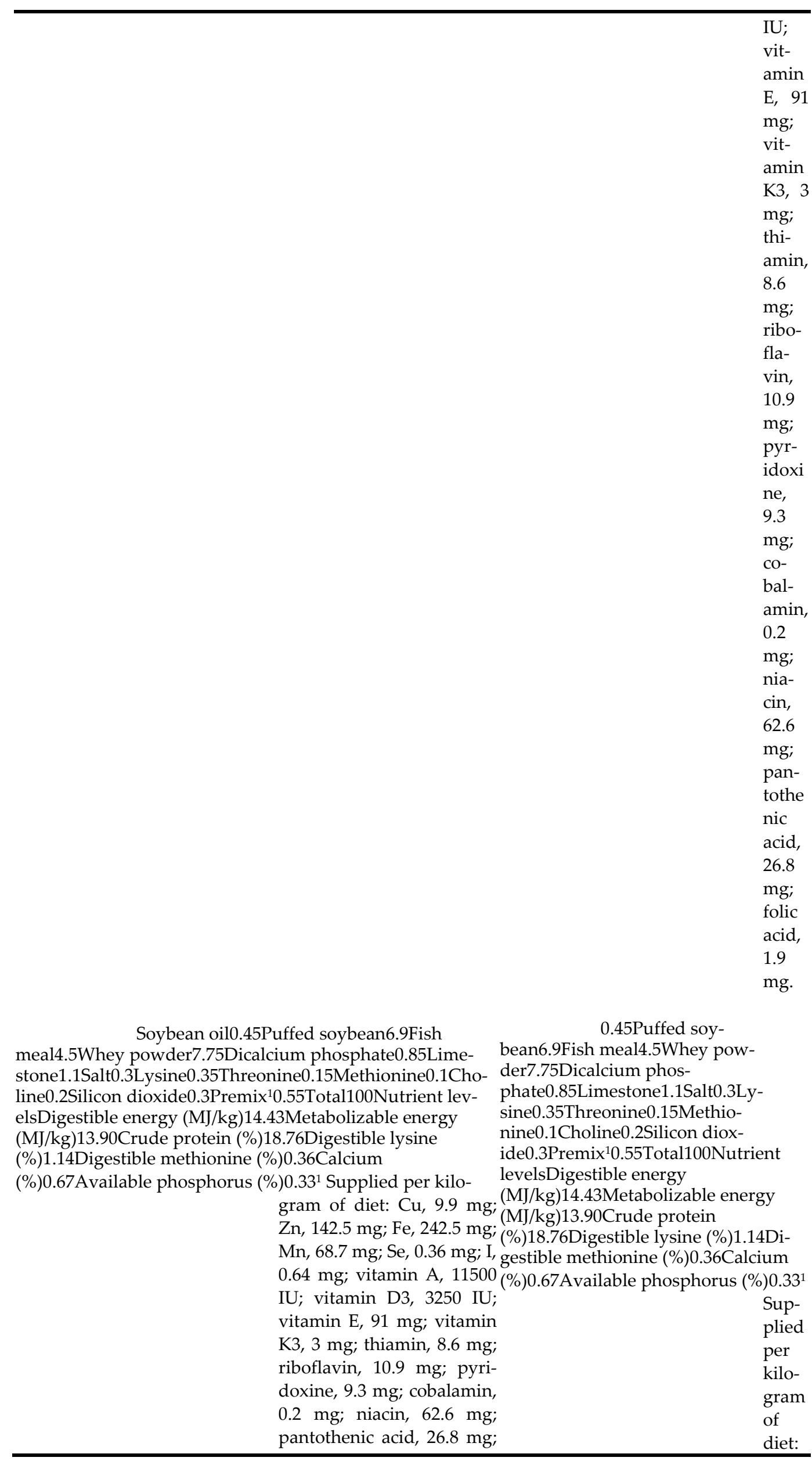


folic acid, $1.9 \mathrm{mg}$. $\mathrm{Cu}$,

$\mathrm{mg}$; I,

0.64

$\mathrm{mg}$;

vitamin A, 11500

IU;

vit-

amin

D3,

3250

IU;

vit-

amin

E, 91

mg;

vit-

amin

K3, 3

mg;

thi-

amin,

8.6

$\mathrm{mg}$;

ribo-

fla-

vin,

10.9

mg;

pyr-

idoxi

ne,

9.3

$\mathrm{mg}$;

CO-

bal-

amin,

0.2

$\mathrm{mg}$;

nia-

cin,

62.6

mg;

pan-

tothe

nic 
Puffed soybean6.9Fish meal4.5Whey powder7.75Dicalcium phosphate0.85Limestone1.1Salt0.3Lysine0.35Threonine0.15Methionine0.1Choline0.2Silicon dioxide0.3Premix ${ }^{1} 0.55$ Total100Nutrient levelsDigestible energy (MJ/kg)14.43Metabolizable energy (MJ/kg)13.90Crude protein (\%)18.76Digestible lysine (\%)1.14Digestible methionine (\%)0.36Calcium (\%)0.67Available phosphorus (\%)0.331 Supplied per kilogram of diet: $\mathrm{Cu}, 9.9 \mathrm{mg}$; Zn, 142.5 $\mathrm{mg}$; $\mathrm{Fe}, 242.5 \mathrm{mg} ; \mathrm{Mn}, 68.7$ $\mathrm{mg}$; Se, $0.36 \mathrm{mg} ; \mathrm{I}, 0.64 \mathrm{mg}$; vitamin A, $11500 \mathrm{IU}$; vitamin D3, 3250 IU; vitamin E, $91 \mathrm{mg}$; vitamin $\mathrm{K} 3,3 \mathrm{mg}$; thiamin, $8.6 \mathrm{mg}$; riboflavin, $10.9 \mathrm{mg}$; pyridoxine, 9.3 $\mathrm{mg}$; cobalamin, $0.2 \mathrm{mg}$; niacin, $62.6 \mathrm{mg}$; pantothenic acid, $26.8 \mathrm{mg}$; folic acid, $1.9 \mathrm{mg}$. acid,

6.9Fish meal4.5Whey powder7.75Dicalcium phosphate0.85Limestone1.1Salt0.3Lysine0.35Threonine0.15Methionine 0.1 Choline 0.2 Silicon dioxide0.3Premix ${ }^{10.55 T o t a l 100 N u t r i e n t}$ levelsDigestible energy (MJ/kg)14.43Metabolizable energy (MJ/kg)13.90Crude protein (\%)18.76Digestible lysine (\%)1.14Digestible methionine (\%)0.36Calcium (\%)0.67Available phosphorus (\%)0.331

Sup-

plied

per

kilo-

gram of diet:

$\mathrm{Cu}$,

9.9

$\mathrm{mg}$;

Zn,

142.5

$\mathrm{mg}$;

$\mathrm{Fe}$,

242.5

$\mathrm{mg}$;

$\mathrm{Mn}$,

68.7

$\mathrm{mg}$;

Se,

0.36

mg; I,

0.64

mg;

vit-

amin

A,

11500

IU;

vit-

amin

D3,

3250

IU;

vit-

amin

E, 91

mg;

vit-

amin

K3, 3 


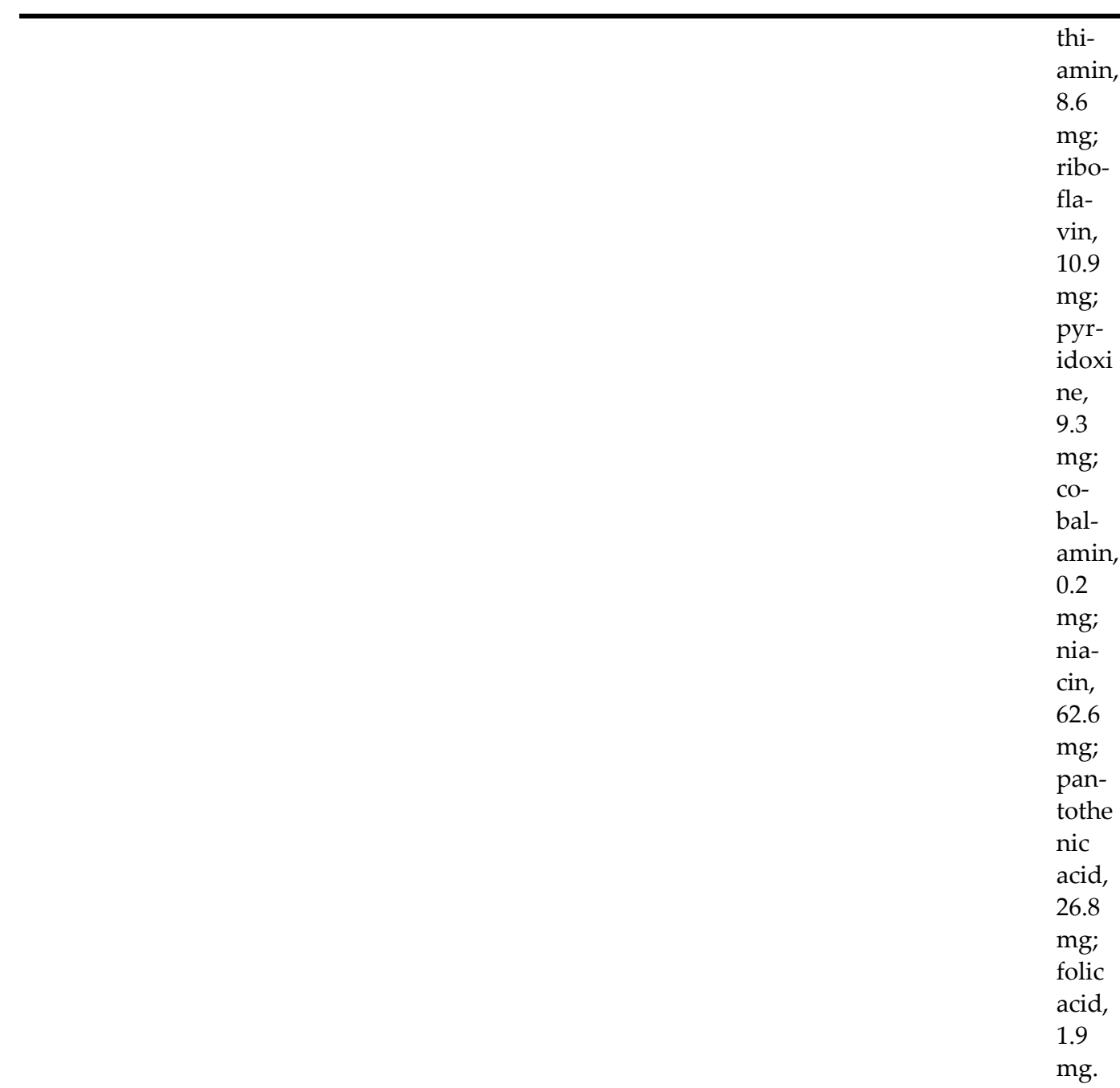

Fish meal4.5Whey powder7.75Dicalcium phosphate0.85Limestone1.1Salt0.3Lysine0.35Threonine0.15Methionine0.1Choline0.2Silicon dioxide0.3Premix ${ }^{1} 0.55$ Total100Nutrient levelsDigestible energy (MJ/kg)14.43Metabolizable energy (MJ/kg)13.90Crude protein (\%)18.76Digestible lysine (\%)1.14Digestible methionine (\%)0.36Calcium (\%)0.67Available phosphorus (\%)0.33 thi-

amin

fla-

vin,

10.9

$\mathrm{mg}$;

pyr-

doxi

$\mathrm{mg}$;

CO-

bal-

amin,

0.2

;

nia-

62.6

$\mathrm{mg}$;

pan-

tothe

nic

cid,

6.8

folic

acid,

$\mathrm{mg}$.

4.5Whey pow-

der7.75Dicalcium phos-

phate0.85Limestone1.1Salt0.3Lysine0.35Threonine0.15Methionine 0.1 Choline 0.2 Silicon dioxide0.3Premix ${ }^{1} 0.55$ Total100Nutrient levelsDigestible energy

(MJ/kg)14.43Metabolizable energy (MJ/kg)13.90Crude protein

(\%)18.76Digestible lysine (\%)1.14Digestible methionine (\%)0.36Calcium Supplied per kilogram of $(\%) 0.67$ Available phosphorus (\%) $0.33^{1}$ diet: $\mathrm{Cu}, 9.9 \mathrm{mg} ; \mathrm{Zn}, 142.5$ $\mathrm{mg}$; Fe, $242.5 \mathrm{mg} ; \mathrm{Mn}, 68.7$ $\mathrm{mg}$; Se, $0.36 \mathrm{mg}$; I, $0.64 \mathrm{mg}$; vitamin A, $11500 \mathrm{IU}$; vitamin D3, 3250 IU; vitamin E, $91 \mathrm{mg}$; vitamin $\mathrm{K} 3,3 \mathrm{mg}$; thiamin, $8.6 \mathrm{mg}$; riboflavin, $10.9 \mathrm{mg}$; pyridoxine, 9.3 $\mathrm{mg}$; cobalamin, $0.2 \mathrm{mg}$; niacin, $62.6 \mathrm{mg}$; pantothenic acid, $26.8 \mathrm{mg}$; folic Supplied per kilogram of diet: $\mathrm{Cu}$, 9.9 mg; Zn, 142.5 


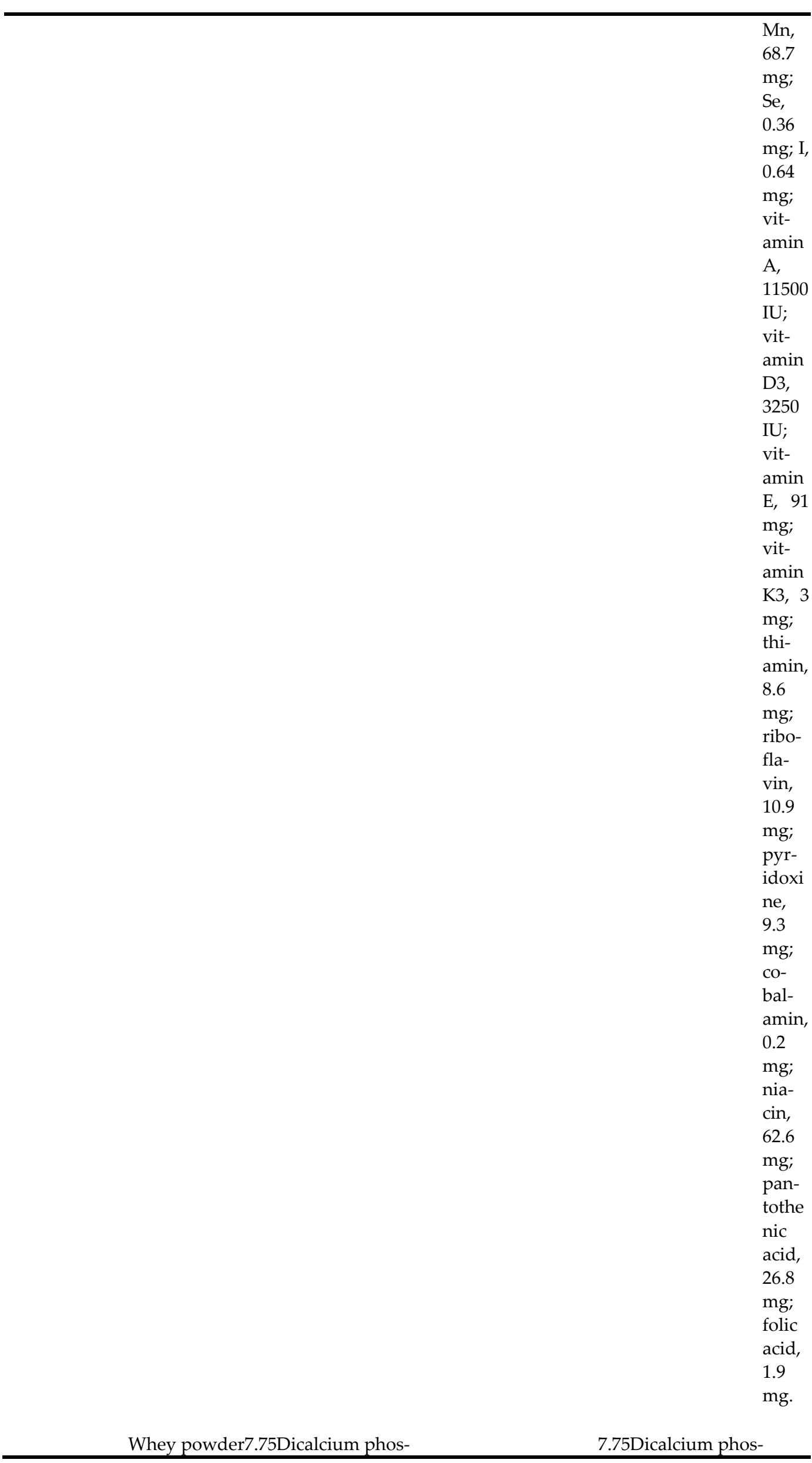


phate0.85Limestone1.1Salt0.3Lysine0.35Threonine0.15Me- phate0.85Limestone1.1Salt0.3Lythionine 0.1 Choline 0.2 Silicon dioxide 0.3 Premix ${ }^{1} 0.55 \mathrm{To}^{-} \quad$ sine 0.35 Threonine0.15Methiotal100Nutrient levelsDigestible energy (MJ/kg)14.43Metab- nine0.1Choline0.2Silicon dioxolizable energy (MJ/kg)13.90Crude protein (\%)18.76Digest- ide0.3Premix ${ }^{1} 0.55$ Total100Nutrient ible lysine (\%)1.14Digestible methionine (\%)0.36Calcium levelsDigestible energy

(\%)0.67Available phosphorus (\%)0.33 ${ }^{1}$ Supplied per kilo- $\quad$ (MJ/kg)14.43Metabolizable energy gram of diet: $\mathrm{Cu}, 9.9 \mathrm{mg}$; (MJ/kg)13.90Crude protein $\mathrm{Zn}, 142.5 \mathrm{mg}$; Fe, $242.5 \mathrm{mg}$; $\%$ )18.76Digestible lysine (\%)1.14Di$\mathrm{Mn}, 68.7 \mathrm{mg}$; Se, $0.36 \mathrm{mg}$; I, gestible methionine (\%)0.36Calcium $0.64 \mathrm{mg}$; vitamin A, $11500(\%) 0.67$ Available phosphorus $(\%) 0.33^{1}$ IU; vitamin D3, 3250 IU; vitamin E, $91 \mathrm{mg}$; vitamin plied $\mathrm{K} 3,3 \mathrm{mg}$; thiamin, $8.6 \mathrm{mg}$; per riboflavin, $10.9 \mathrm{mg}$; pyridoxine, $9.3 \mathrm{mg}$; cobalamin, $0.2 \mathrm{mg}$; niacin, $62.6 \mathrm{mg}$; pantothenic acid, $26.8 \mathrm{mg}$; folic acid, $1.9 \mathrm{mg}$.

kilogram of diet: $\mathrm{Cu}$, 9.9 mg; Zn, 142.5 $\mathrm{mg}$; $\mathrm{Fe}$, 242.5

$\mathrm{mg}$;

$\mathrm{Mn}$,

68.7

mg;

Se,

0.36

$\mathrm{mg}$; I,

0.64

mg;

vitamin

A, 11500

IU;

vit-

amin

D3,

3250

IU;

vit-

amin

E, 91

mg;

vit-

amin

K3, 3

$\mathrm{mg}$;

thi-

amin,

8.6

mg;

ribo-

fla-

vin,

10.9

$\mathrm{mg}$; 


\begin{tabular}{|c|c|}
\hline 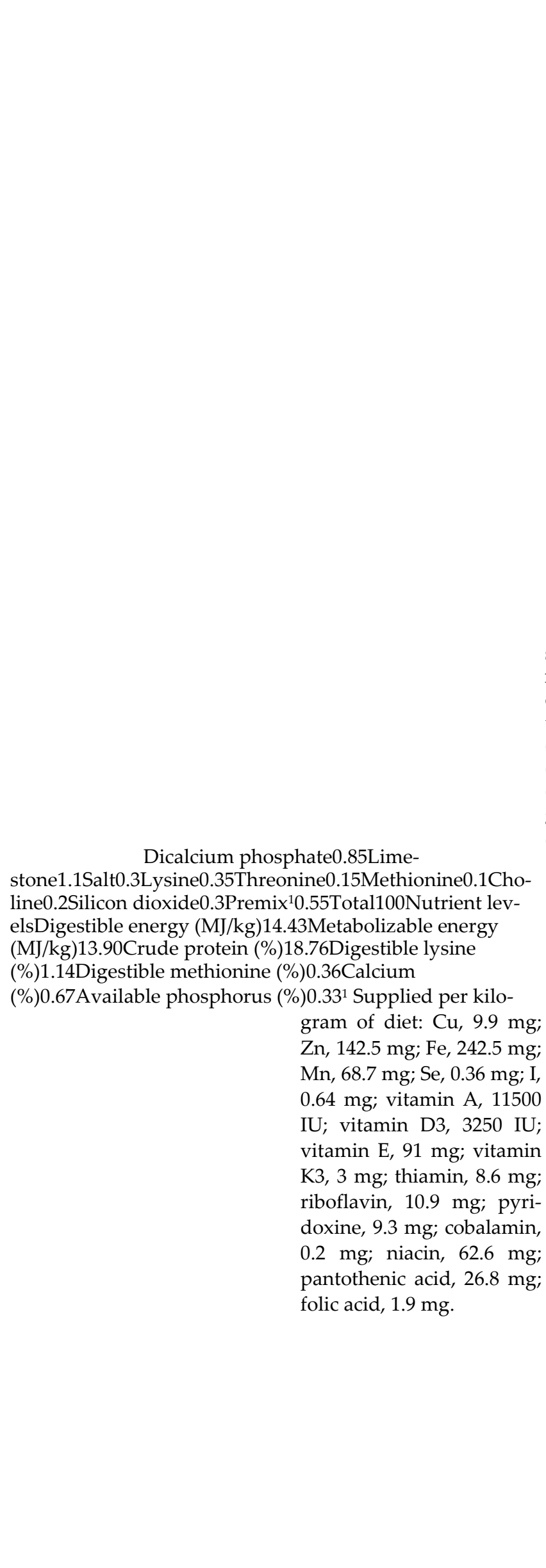 & 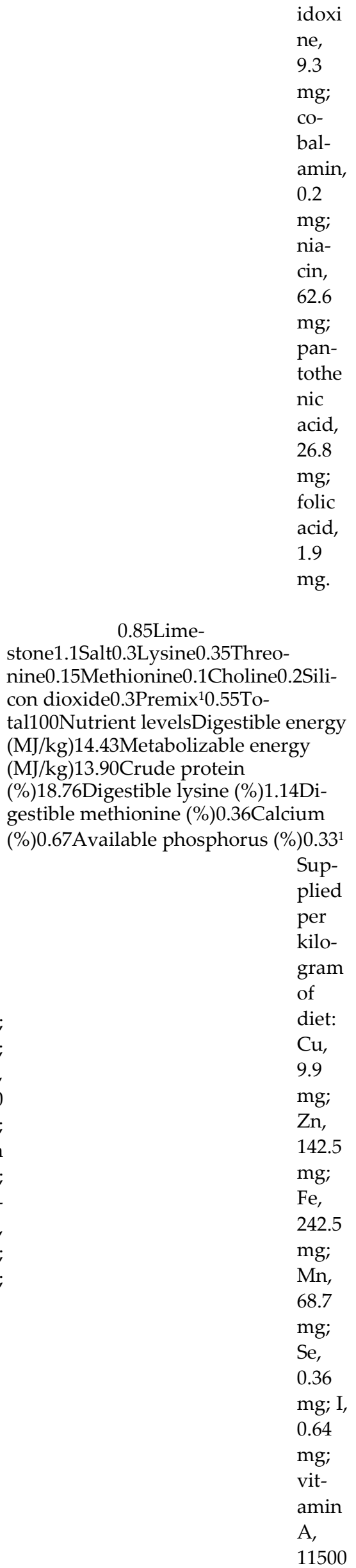 \\
\hline
\end{tabular}




\begin{tabular}{|c|c|}
\hline & $\begin{array}{l}\text { IU; } \\
\text { vit- } \\
\text { amin } \\
\text { D3, } \\
3250 \\
\text { IU; } \\
\text { vit- } \\
\text { amin } \\
\text { E, 91 } \\
\text { mg; } \\
\text { vit- } \\
\text { amin } \\
\text { K3, } 3 \\
\text { mg; } \\
\text { thi- } \\
\text { amin, } \\
8.6 \\
\text { mg; } \\
\text { ribo- } \\
\text { fla- } \\
\text { vin, } \\
10.9 \\
\text { mg; } \\
\text { pyr- } \\
\text { idoxi } \\
\text { ne, } \\
9.3 \\
\text { mg; } \\
\text { co- } \\
\text { bal- } \\
\text { amin, } \\
0.2 \\
\text { mg; } \\
\text { nia- } \\
\text { cin, } \\
62.6 \\
\text { mg; } \\
\text { pan- } \\
\text { tothe } \\
\text { nic } \\
\text { acid, } \\
26.8 \\
\text { mg; } \\
\text { folic } \\
\text { aid, }\end{array}$ \\
\hline \multicolumn{2}{|c|}{ 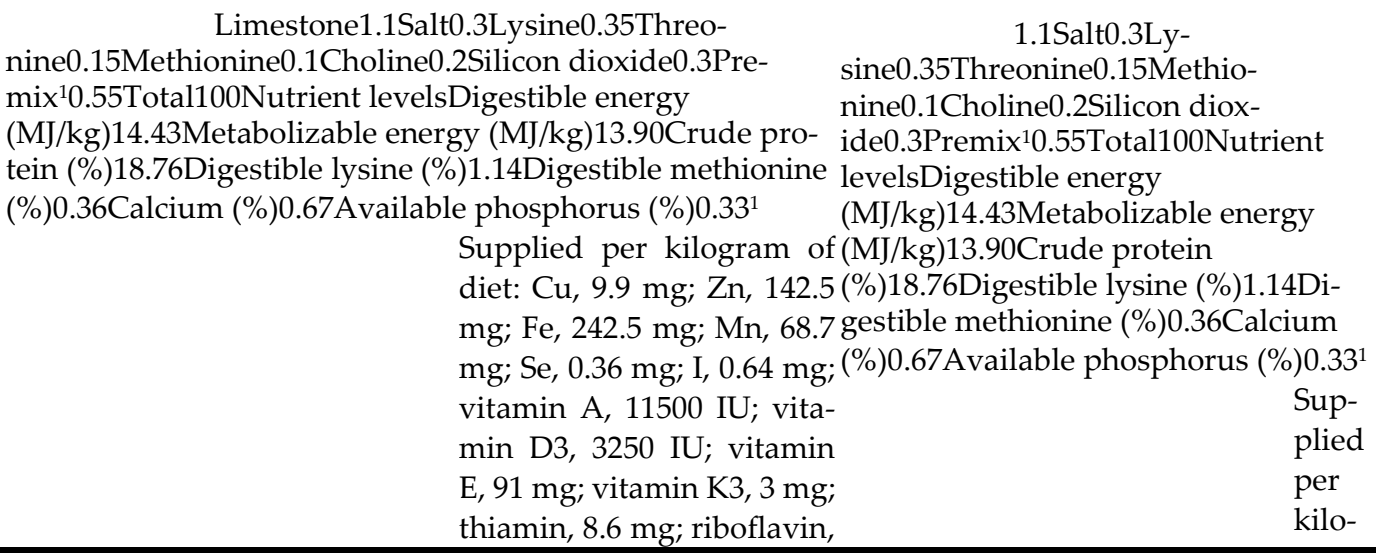 } \\
\hline
\end{tabular}


$10.9 \mathrm{mg} ;$ pyridoxine, 9.3

$\mathrm{mg}$; cobalamin, $0.2 \mathrm{mg}$;

niacin, $62.6 \mathrm{mg}$; panto-

gram

thenic acid, $26.8 \mathrm{mg}$; folic

of

acid, $1.9 \mathrm{mg}$.

diet:

$\mathrm{Cu}$,

9.9

mg;

Zn,

142.5

$\mathrm{mg}$;

$\mathrm{Fe}$,

242.5

$\mathrm{mg}$;

$\mathrm{Mn}$,

68.7

$\mathrm{mg}$;

Se,

0.36

$\mathrm{mg}$; I,

0.64

$\mathrm{mg}$;

vit-

amin

A,

11500

IU;

vit-

amin

D3,

3250

IU;

vit-

amin

E, 91

mg;

vit-

amin

K3, 3

$\mathrm{mg}$;

thi-

amin,

8.6

mg;

ribo-

fla-

vin,

10.9

mg;

pyr-

idoxi

ne,

9.3

$\mathrm{mg}$;

CO-

bal-

amin,

0.2

$\mathrm{mg}$;

nia-

cin,

62.6 
Salt0.3Lysine0.35Threonine0.15Methionine 0.1 Choline 0.2 Silicon dioxide 0.3 Premix ${ }^{1} 0.55$ Total100Nutrient levelsDigestible energy (MJ/kg)14.43Metabolizable energy (MJ/kg)13.90Crude protein (\%)18.76Digestible lysine (\%)1.14Digestible methionine (\%)0.36Calcium (\%)0.67Available phosphorus (\%)0.33 ${ }^{1}$ Supplied per kilogram of diet: $\mathrm{Cu}, 9.9 \mathrm{mg}$; $\mathrm{Zn}, 142.5 \mathrm{mg}$; Fe, $242.5 \mathrm{mg}$; $\mathrm{Mn}, 68.7 \mathrm{mg}$; Se, $0.36 \mathrm{mg}$; I, $0.64 \mathrm{mg}$; vitamin A, 11500 IU; vitamin D3, 3250 IU;

0.3Lysine 0.35 Threonine0.15Methionine0.1Choline0.2Silicon dioxide 0.3 Premix ${ }^{1} 0.55$ Total100Nutrient levelsDigestible energy (MJ/kg)14.43Metabolizable energy (MJ/kg)13.90Crude protein

(\%)18.76Digestible lysine (\%)1.14Digestible methionine (\%)0.36Calcium (\%)0.67Available phosphorus (\%)0.33 ${ }^{1}$

Supplied per kilogram of diet: $\mathrm{Cu}$, 9.9 $\mathrm{mg}$;

$\mathrm{Zn}$,

142.5

$\mathrm{mg}$;

$\mathrm{Fe}$, 242.5

$\mathrm{mg}$;

$\mathrm{Mn}$,

68.7 vitamin E, $91 \mathrm{mg}$; vitamin $\mathrm{mg}$; $\mathrm{K} 3,3 \mathrm{mg}$; thiamin, $8.6 \mathrm{mg}$; riboflavin, $10.9 \mathrm{mg}$; pyridoxine, $9.3 \mathrm{mg}$; cobalamin, Se, 0.36 $\mathrm{mg}$; I, $0.2 \mathrm{mg}$; niacin, $62.6 \mathrm{mg}$; pantothenic acid, $26.8 \mathrm{mg}$; 0.64

mg;

vitamin A, 11500

IU;

vit-

amin

D3,

3250

IU;

vit-

amin

E, 91

mg;

vit-

amin

$\mathrm{K} 3,3$ 


\begin{tabular}{|c|c|}
\hline 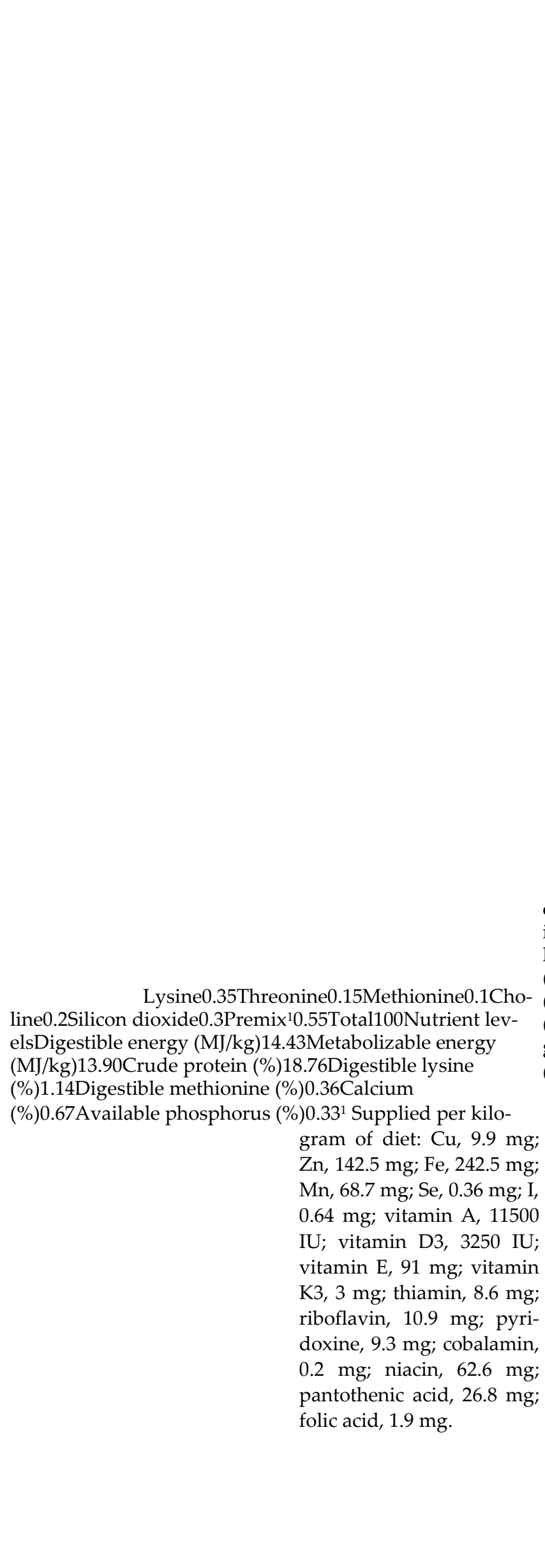 & 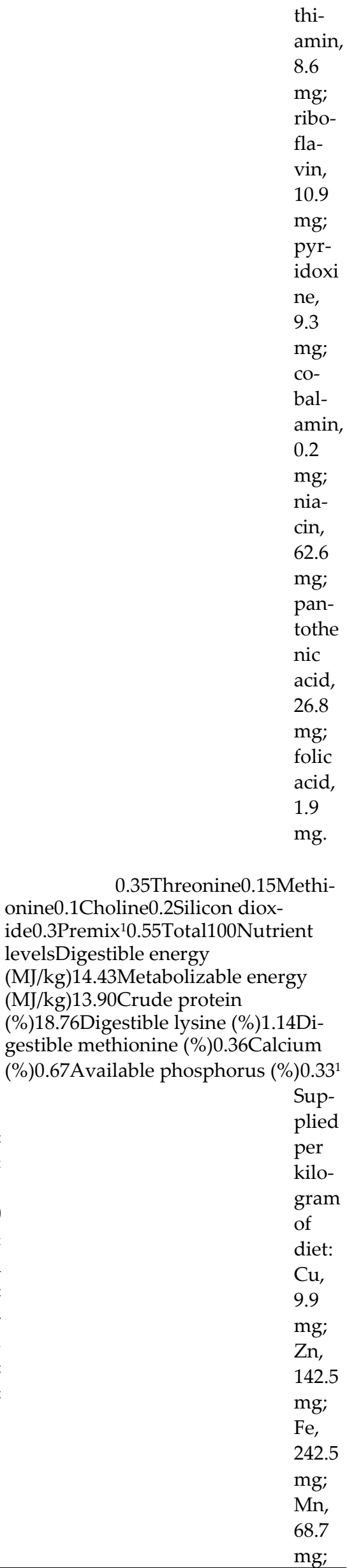 \\
\hline
\end{tabular}




\begin{tabular}{|c|c|}
\hline & $\begin{array}{l}\text { Se, } \\
0.36 \\
\text { mg; I, } \\
0.64 \\
\text { mg; } \\
\text { vit- } \\
\text { amin } \\
\text { A, } \\
\text { 11500 } \\
\text { IU; } \\
\text { vit- } \\
\text { amin } \\
\text { D3, } \\
3250 \\
\text { IU; } \\
\text { vit- } \\
\text { amin } \\
\text { E, 91 } \\
\text { mg; } \\
\text { vit- } \\
\text { amin } \\
\text { K3, } 3 \\
\text { mg; } \\
\text { thi- } \\
\text { amin, } \\
8.6 \\
\text { mg; } \\
\text { ribo- } \\
\text { fla- } \\
\text { vin, } \\
10.9 \\
\text { mg; } \\
\text { pyr- } \\
\text { idoxi } \\
\text { ne, } \\
9.3 \\
\text { mg; } \\
\text { co- } \\
\text { bal- } \\
\text { amin, } \\
0.2 \\
\text { mg; } \\
\text { nia- } \\
\text { cin, } \\
62.6 \\
\text { mg; } \\
\text { pan- } \\
\text { tothe } \\
\text { nic } \\
\text { acid, } \\
26.8 \\
\text { mg; } \\
\text { folic } \\
\text { acid, } \\
\text { m.9 } \\
\text { mg. }\end{array}$ \\
\hline \multicolumn{2}{|c|}{$\begin{array}{lc}\text { Threonine0.15Methionine0.1Choline0.2Sili- } & 0.15 \mathrm{Methionine0.1Cho-} \\
\text { con dioxide0.3Premix }{ }^{1} 0.55 T \text { Total100Nutrient levelsDigestible line0.2Silicon dioxide0.3Pre- } \\
\text { energy (MJ/kg)14.43Metabolizable energy } & \text { mix } 10.55 \text { Total100Nutrient levelsDi- } \\
\text { (MJ/kg)13.90Crude protein (\%)18.76Digestible lysine } & \text { gestible energy (MJ } / \mathrm{kg}) 14.43 \mathrm{Metabo-} \\
(\%) 1.14 \text { Digestible methionine (\%)0.36Calcium } & \text { lizable energy (MJ } / \mathrm{kg}) 13.90 \mathrm{Crude} \\
\end{array}$} \\
\hline
\end{tabular}


(\%)0.67Available phosphorus (\%)0.33 ${ }^{1}$ Supplied per kilo- $\quad$ protein (\%)18.76Digestible lysine gram of diet: $\mathrm{Cu}, 9.9 \mathrm{mg}$; $(\%) 1.14$ Digestible methionine $\mathrm{Zn}, 142.5 \mathrm{mg}$; Fe, $242.5 \mathrm{mg}$; $(\%) 0.36$ Calcium (\%)0.67Available $\mathrm{Mn}, 68.7 \mathrm{mg}$; Se, $0.36 \mathrm{mg}$; I, phosphorus (\%)0.33 ${ }^{1}$ Supplied per $0.64 \mathrm{mg}$; vitamin A, 11500

kiloIU; vitamin D3, 3250 IU; gram vitamin E, $91 \mathrm{mg}$; vitamin of $\mathrm{K} 3,3 \mathrm{mg}$; thiamin, $8.6 \mathrm{mg}$; riboflavin, $10.9 \mathrm{mg}$; pyridoxine, $9.3 \mathrm{mg}$; cobalamin, diet: $0.2 \mathrm{mg}$; niacin, $62.6 \mathrm{mg}$; $\mathrm{Cu}$, pantothenic acid, $26.8 \mathrm{mg}$; 9.9 $\mathrm{mg}$; folic acid, $1.9 \mathrm{mg}$.

$\mathrm{Zn}$,

142.5

$\mathrm{mg}$;

$\mathrm{Fe}$,

242.5

$\mathrm{mg}$;

$\mathrm{Mn}$,

68.7

$\mathrm{mg}$;

Se,

0.36

$\mathrm{mg}$; I,

0.64

$\mathrm{mg}$;

vit-

amin

A,

IU;

vit-

amin

D3,

3250

IU;

vit-

amin

E, 91

mg;

vit-

amin

$\mathrm{K} 3,3$

$\mathrm{mg}$;

thi-

amin,

8.6

$\mathrm{mg}$;

ribo-

fla-

vin,

10.9

$\mathrm{mg}$;

pyr-

idoxi

ne,

9.3

$\mathrm{mg}$;

CO-

bal-

amin, 
Methionine0.1Choline0.2Silicon diox-

ide0.3Premix ${ }^{1} 0.55$ Total100Nutrient levelsDigestible energy $(\mathrm{MJ} / \mathrm{kg}) 14.43 \mathrm{Metabolizable} \mathrm{energy} \mathrm{(MJ/kg)13.90Crude} \mathrm{pro-}$ tein (\%)18.76Digestible lysine (\%)1.14Digestible methionine (\%)0.36Calcium (\%)0.67Available phosphorus (\%)0.331

Supplied per kilogram of diet: $\mathrm{Cu}, 9.9 \mathrm{mg} ; \mathrm{Zn}, 142.5$ $\mathrm{mg}$; Fe, $242.5 \mathrm{mg} ; \mathrm{Mn}, 68.7$ $\mathrm{mg}$; Se, $0.36 \mathrm{mg}$; I, $0.64 \mathrm{mg}$; vitamin A, $11500 \mathrm{IU}$; vitamin D3, $3250 \mathrm{IU}$; vitamin

0.1Choline 0.2 Silicon dioxide 0.3 Premix ${ }^{1} 0.55$ Total100Nutrient levelsDigestible energy

(MJ/kg)14.43Metabolizable energy

(MJ/kg)13.90Crude protein

(\%)18.76Digestible lysine (\%)1.14Digestible methionine (\%)0.36Calcium (\%)0.67Available phosphorus (\%)0.331

Sup-

plied

per

kilo-

gram

of

diet:

$\mathrm{Cu}$,

9.9

$\mathrm{mg}$;

Zn,

142.5

$\mathrm{mg}$;

$\mathrm{Fe}$,

242.5

$\mathrm{mg}$;

$\mathrm{Mn}$,

E, $91 \mathrm{mg}$; vitamin $\mathrm{K} 3,3 \mathrm{mg}$;

68.7 thiamin, $8.6 \mathrm{mg}$; riboflavin,

$\mathrm{mg}$;

$10.9 \mathrm{mg}$; pyridoxine, 9.3

Se,

$\mathrm{mg}$; cobalamin, $0.2 \mathrm{mg}$;

0.36

niacin, $62.6 \mathrm{mg}$; panto-

mg; I,

thenic acid, $26.8 \mathrm{mg}$; folic

0.64

acid, $1.9 \mathrm{mg}$.

$\mathrm{mg}$;

vit-

amin

A,

IU;

vit-

amin

D3,

3250

IU;

vit-

amin

E, 91

mg; 


\begin{tabular}{|c|c|}
\hline 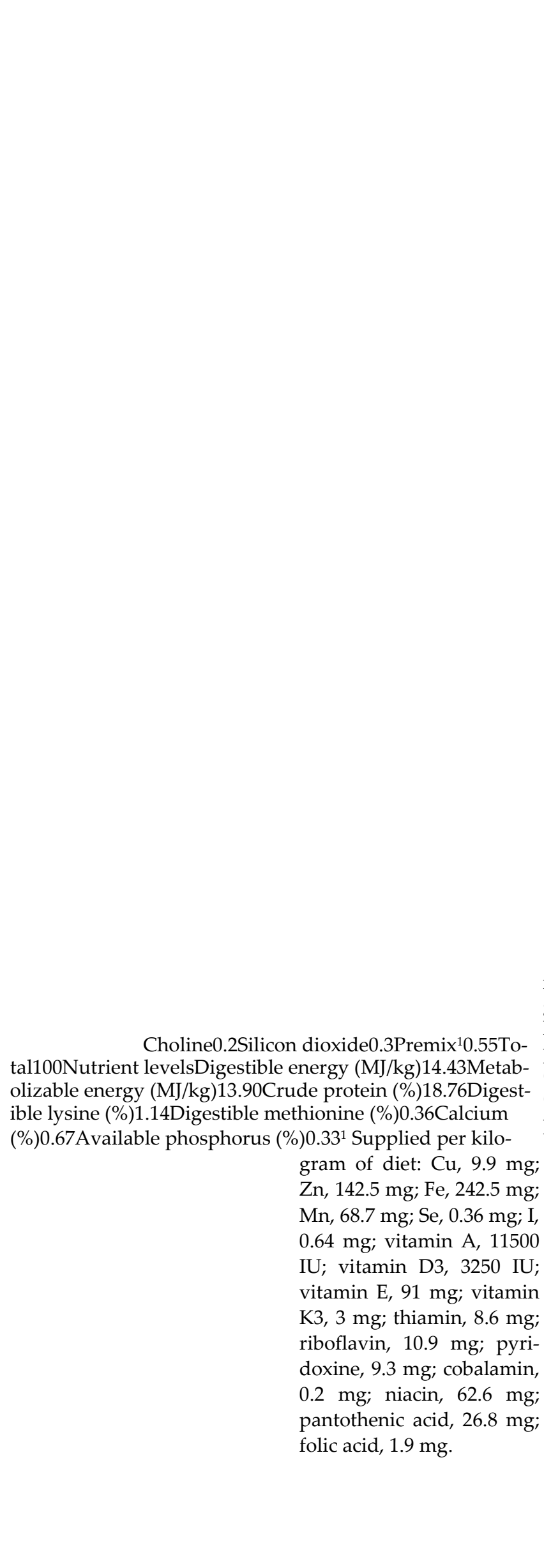 & 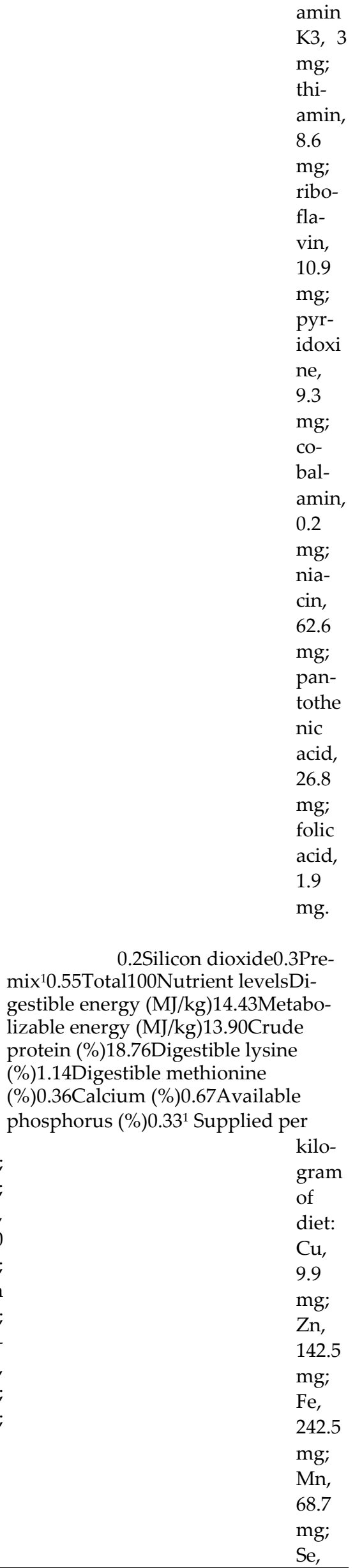 \\
\hline
\end{tabular}




\begin{tabular}{|c|c|}
\hline & $\begin{array}{l}0.36 \\
\text { mg; I, } \\
0.64 \\
\text { mg; } \\
\text { vit- } \\
\text { amin } \\
\text { A, } \\
11500 \\
\text { IU; } \\
\text { vit- } \\
\text { amin } \\
\text { D3, } \\
3250 \\
\text { IU; } \\
\text { vit- } \\
\text { amin } \\
\text { E, } 91 \\
\text { mg; } \\
\text { vit- } \\
\text { amin } \\
\text { K3, } 3 \\
\text { mg; } \\
\text { thi- } \\
\text { amin, } \\
8.6 \\
\text { mg; } \\
\text { ribo- } \\
\text { fla- } \\
\text { vin, } \\
10.9 \\
\text { mg; } \\
\text { pyr- } \\
\text { idoxi } \\
\text { ne, } \\
9.3 \\
\text { mg; } \\
\text { co- } \\
\text { bal- } \\
\text { amin, } \\
0.2 \\
\text { mg; } \\
\text { nia- } \\
\text { cin, } \\
62.6 \\
\text { mg; } \\
\text { pan- } \\
\text { tothe } \\
\text { nic } \\
\text { acid, } \\
26.8 \\
\text { mg; } \\
\text { folic } \\
\text { acid, } \\
\text { mg. }\end{array}$ \\
\hline 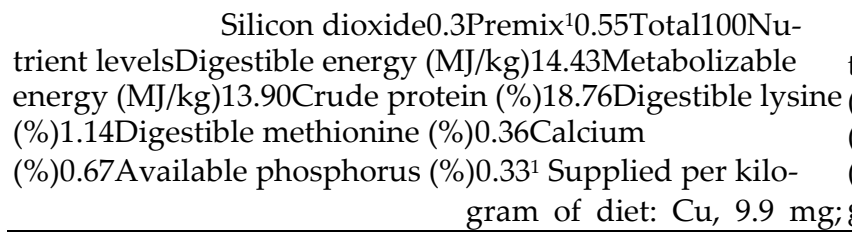 & $\begin{array}{l}\text { 0.3Premix }{ }^{1} 0.55 \text { To- } \\
\text { tal100Nutrient levelsDigestible energy } \\
(\mathrm{MJ} / \mathrm{kg}) 14.43 \text { Metabolizable energy } \\
\text { (MJ/kg)13.90Crude protein } \\
\text { (\%)18.76Digestible lysine (\%)1.14Di- } \\
\text { gestible methionine (\%)0.36Calcium }\end{array}$ \\
\hline
\end{tabular}


$\mathrm{Zn}, 142.5 \mathrm{mg}$; Fe, $242.5 \mathrm{mg}$; $\%$ )0.67Available phosphorus (\%)0.33 $\mathrm{Mn}, 68.7 \mathrm{mg}$; Se, $0.36 \mathrm{mg}$; I, $0.64 \mathrm{mg}$; vitamin A, 11500 IU; vitamin D3, 3250 IU; vitamin E, $91 \mathrm{mg}$; vitamin $\mathrm{K} 3,3 \mathrm{mg}$; thiamin, $8.6 \mathrm{mg}$; riboflavin, $10.9 \mathrm{mg}$; pyridoxine, $9.3 \mathrm{mg}$; cobalamin, $0.2 \mathrm{mg}$; niacin, $62.6 \mathrm{mg}$; pantothenic acid, $26.8 \mathrm{mg}$; folic acid, $1.9 \mathrm{mg}$.

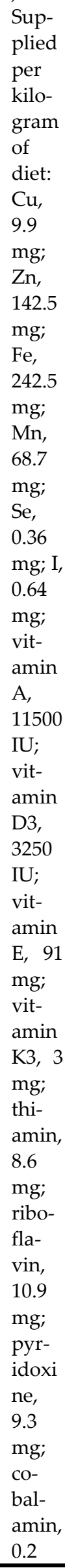




\begin{tabular}{|c|c|}
\hline $\begin{array}{l}\text { Premix }{ }^{1} \text { 0.55Total100Nutrient levelsDigesti- } \\
\text { ble energy (MJ/kg)14.43Metabolizable energy } \\
\text { (MJ/kg)13.90Crude protein (\%)18.76Digestible lysine } \\
\text { (\%)1.14Digestible methionine (\%)0.36Calcium } \\
\text { (\%)0.67Available phosphorus (\%) } 0.33^{1} \text { Supplied per kilo- } \\
\text { gram of diet: Cu, } 9.9 \mathrm{mg} ; \\
\text { Zn, } 142.5 \mathrm{mg} \text {; Fe, } 242.5 \mathrm{mg} ; \\
\text { Mn, } 68.7 \mathrm{mg} \text {; Se, } 0.36 \mathrm{mg} \text {; I, } \\
0.64 \mathrm{mg} \text {; vitamin A, } 11500 \\
\text { IU; vitamin D3, } 3250 \mathrm{IU} ; \\
\text { vitamin E, } 91 \mathrm{mg} ; \text { vitamin } \\
\text { K3, 3 mg; thiamin, } 8.6 \mathrm{mg} ; \\
\text { riboflavin, } 10.9 \mathrm{mg} ; \mathrm{pyri}- \\
\text { doxine, } 9.3 \mathrm{mg} \text { cobalamin, } \\
0.2 \mathrm{mg} \text { niacin, } 62.6 \mathrm{mg} ; \\
\text { pantothenic acid, } 26.8 \mathrm{mg} ; \\
\text { folic acid, } 1.9 \mathrm{mg} .\end{array}$ & 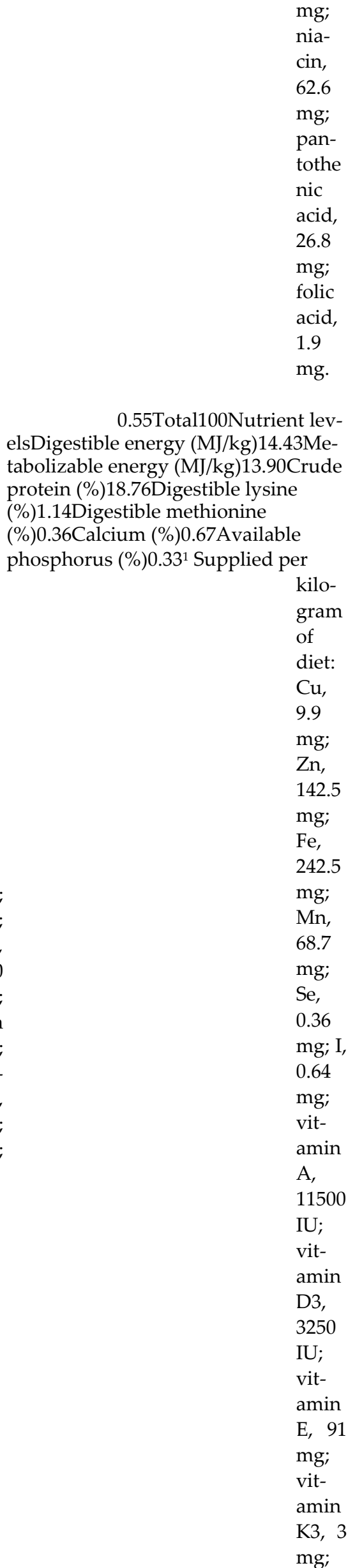 \\
\hline
\end{tabular}




thi-
amin,
8.6
mg;
ribo-
fla-
vin,
10.9
mg;
pyr-
idoxi
ne,
9.3
mg;
co-
bal-
amin,
0.2
mg;
nia-
cin,
62.6
mg;
pan-
tothe
nic
acid,
26.8
mg;
folic
acid,
1.9
mg.

100Nutrient levelsDigestible energy (MJ/kg)14.43Metabolizable energy (MJ/kg)13.90Crude protein (\%)18.76Digestible lysine

Total100Nutrient levelsDigestible energy (\%)1.14Digestible methionine (\%)0.36Calcium (\%)0.67Available (MJ/kg)14.43Metabolizable energy (MJ/kg)13.90Crude pro- phosphorus (\%)0.33 ${ }^{1}$ Supplied per tein (\%)18.76Digestible lysine (\%)1.14Digestible methionine kilo(\%)0.36Calcium (\%)0.67Available phosphorus (\%)0.331

Supplied per kilogram of diet: $\mathrm{Cu}, 9.9 \mathrm{mg} ; \mathrm{Zn}, 142.5$ $\mathrm{mg} ; \mathrm{Fe}, 242.5 \mathrm{mg} ; \mathrm{Mn}, 68.7$ $\mathrm{mg}$; Se, $0.36 \mathrm{mg}$; I, $0.64 \mathrm{mg}$; vitamin A, 11500 IU; vitamin D3, 3250 IU; vitamin E, $91 \mathrm{mg}$; vitamin $\mathrm{K} 3,3 \mathrm{mg}$; thiamin, $8.6 \mathrm{mg}$; riboflavin, $10.9 \mathrm{mg}$; pyridoxine, 9.3 $\mathrm{mg}$; cobalamin, $0.2 \mathrm{mg}$; gram of diet: $\mathrm{Cu}$, 9.9 $\mathrm{mg}$; $\mathrm{Zn}$, 142.5 $\mathrm{mg}$; $\mathrm{Fe}$, niacin, $62.6 \mathrm{mg}$; panto242.5 thenic acid, $26.8 \mathrm{mg}$; folic $\mathrm{mg}$; acid, $1.9 \mathrm{mg}$. 


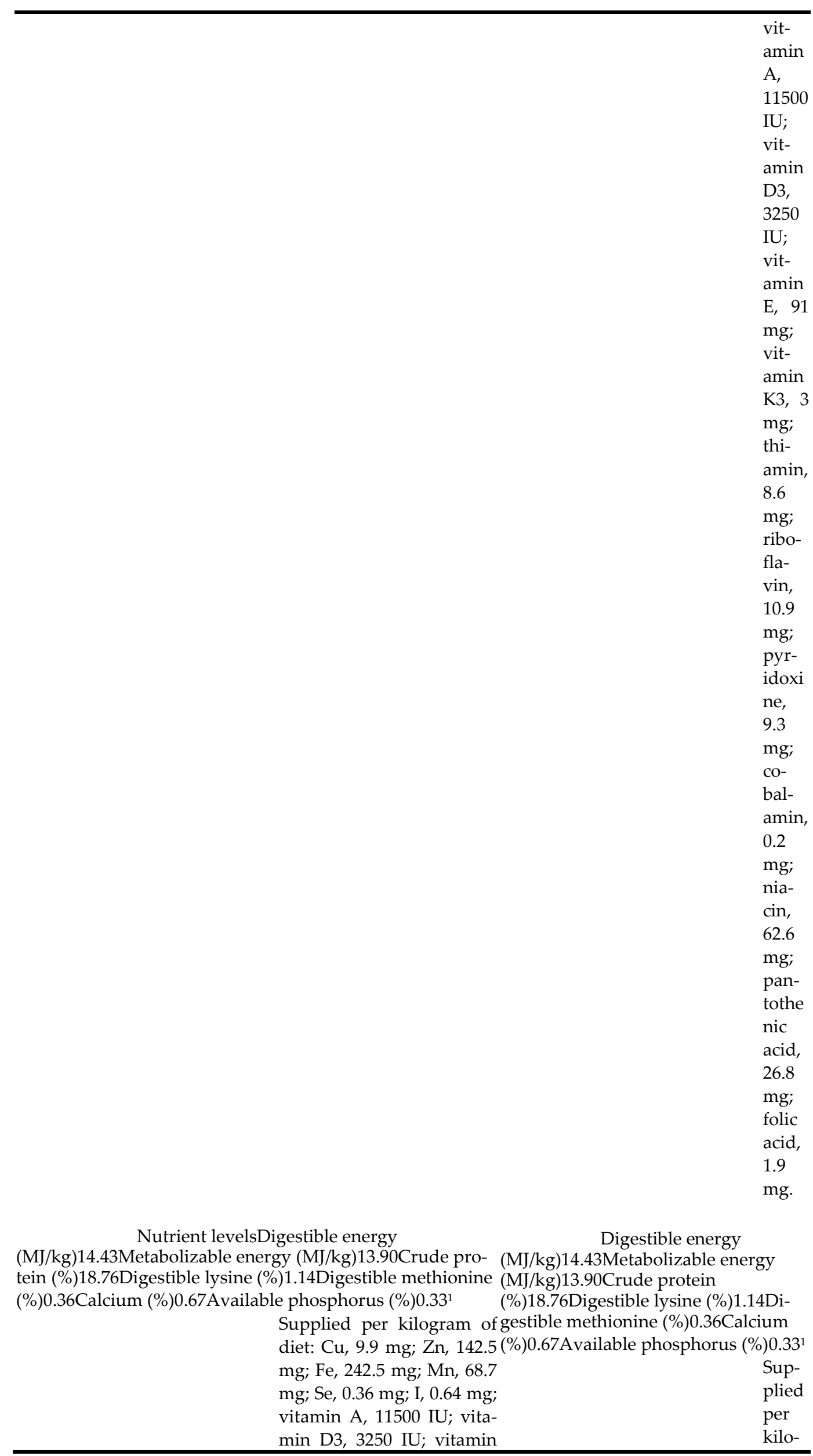


E, $91 \mathrm{mg}$; vitamin $\mathrm{K} 3,3 \mathrm{mg}$; thiamin, $8.6 \mathrm{mg}$; riboflavin, gram $10.9 \mathrm{mg}$; pyridoxine, 9.3 of $\mathrm{mg}$; cobalamin, $0.2 \mathrm{mg}$; niacin, $62.6 \mathrm{mg}$; pantodiet: thenic acid, $26.8 \mathrm{mg}$; folic $\mathrm{Cu}$, 9.9 acid, $1.9 \mathrm{mg}$. 


pan-
tothe
nic
acid,
26.8
mg;
folic
acid,
1.9
mg.

14.43Metabolizable energy $(\mathrm{MJ} / \mathrm{kg}) 13.90$ Crude protein (\%)18.76Digestible lysine (\%)1.14Digestible methionine (\%)0.36Calcium (\%)0.67Available phosphorus (\%) $0.33^{1}$

Sup-
plied
per
kilo-
gram
of
diet:
Cu,
9.9
mg;
Zn,
142.5
mg;
Fe,
242.5
mg;
Mn,
68.7
mg;
Se,
0.36
mg; I,
0.64
mg;
vit-
amin
A,
11500
IU;
vit-
amin
D3,
3250
IU;
vit-
amin
E, 91
mg;
vit-
amin
K3, 3
mg;

energy (MJ/kg)13.90Crude protein (\%)18.76Digestible lysine (\%)1.14Digestible methionine (\%)0.36Calcium

(\%)0.67Available phosphorus (\%)0.33 ${ }^{1}$ Supplied per kilogram of diet: $\mathrm{Cu}, 9.9 \mathrm{mg}$; $\mathrm{Zn}, 142.5 \mathrm{mg}$; Fe, $242.5 \mathrm{mg}$; $\mathrm{Mn}, 68.7 \mathrm{mg}$; Se, $0.36 \mathrm{mg}$; I, $0.64 \mathrm{mg}$; vitamin A, 11500 IU; vitamin D3, 3250 IU; vitamin E, $91 \mathrm{mg}$; vitamin $\mathrm{K} 3,3 \mathrm{mg}$; thiamin, $8.6 \mathrm{mg}$; riboflavin, $10.9 \mathrm{mg}$; pyridoxine, $9.3 \mathrm{mg}$; cobalamin, $0.2 \mathrm{mg}$; niacin, $62.6 \mathrm{mg}$; pantothenic acid, $26.8 \mathrm{mg}$; folic acid, $1.9 \mathrm{mg}$. 


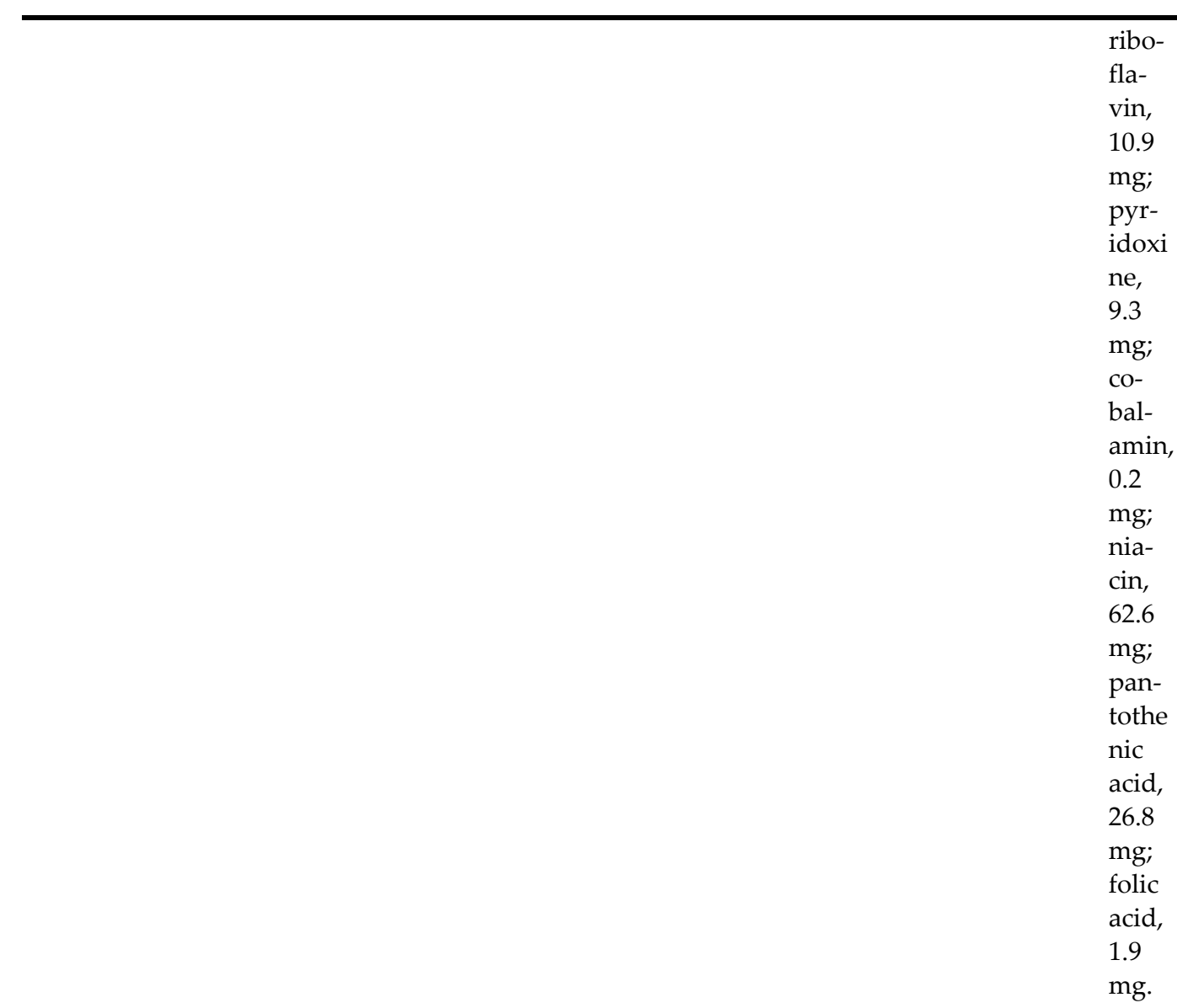

13.90Crude protein (\%)18.76Digestible lysine (\%)1.14Digestible methionine (\%)0.36Calcium (\%)0.67Available phosphorus (\%) $0.33^{1}$

Sup-

plied

per

kilo-

gram

of

diet:

$\mathrm{Cu}$,

9.9

$\mathrm{mg}$;

$\mathrm{Zn}$,

Metabolizable energy (MJ/kg)13.90Crude

142.5

protein (\%)18.76Digestible lysine (\%)1.14Digestible methio-

mg;

nine (\%)0.36Calcium (\%)0.67Available phosphorus (\%)0.331

$\mathrm{Fe}$,

Supplied per kilogram of

diet: $\mathrm{Cu}, 9.9 \mathrm{mg}$; Zn, 142.5

$\mathrm{mg}$;

$\mathrm{mg}$; Fe, 242.5 mg; Mn, 68.7

$\mathrm{Mn}$,

$\mathrm{mg}$; Se, $0.36 \mathrm{mg}$; I, $0.64 \mathrm{mg}$;

68.7

vitamin A, 11500 IU; vita-

$\mathrm{mg}$;

min D3, 3250 IU; vitamin

Se,

E, $91 \mathrm{mg}$; vitamin $\mathrm{K} 3,3 \mathrm{mg}$;

0.36 thiamin, $8.6 \mathrm{mg}$; riboflavin,

mg; I,

$10.9 \mathrm{mg}$; pyridoxine, 9.3

0.64

$\mathrm{mg}$; cobalamin, $0.2 \mathrm{mg}$;

mg;

niacin, $62.6 \mathrm{mg}$; panto-

vit-

thenic acid, $26.8 \mathrm{mg}$; folic

amin

acid, $1.9 \mathrm{mg}$.

A, 


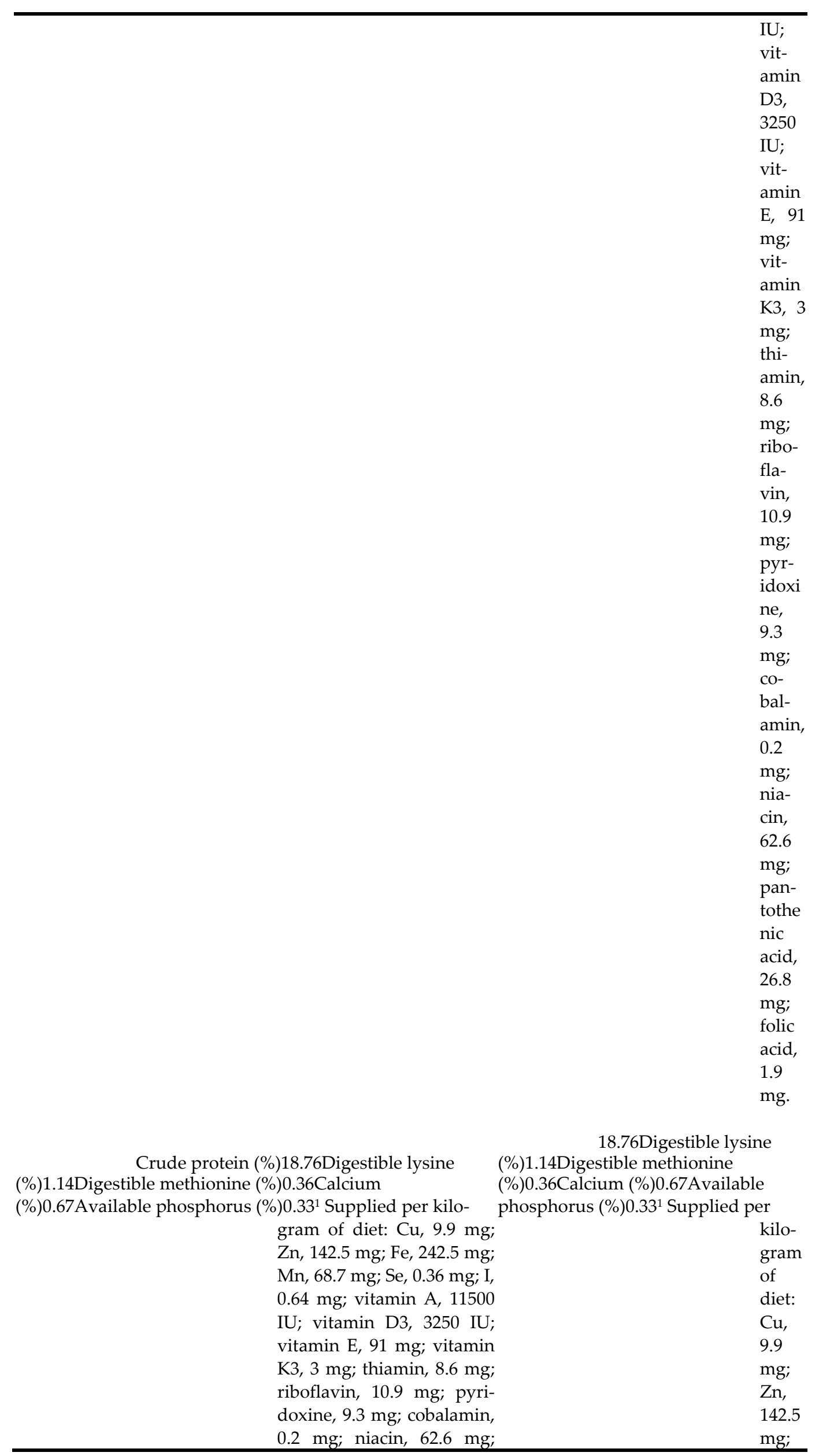


pantothenic acid, $26.8 \mathrm{mg}$;

folic acid, $1.9 \mathrm{mg}$.

242.5

$\mathrm{mg}$;

$\mathrm{Mn}$,

68.7

$\mathrm{mg}$;

Se,

0.36

mg; I,

0.64

$\mathrm{mg}$;

vit-

amin

A,

11500

IU;

vit-

amin

D3,

3250

IU;

vit-

amin

E, 91

mg;

vit-

amin

K3, 3

$\mathrm{mg}$;

thi-

amin,

8.6

$\mathrm{mg}$;

ribo-

fla-

vin,

10.9

$\mathrm{mg}$;

pyr-

idoxi

ne,

9.3

mg;

CO-

bal-

amin,

0.2

mg;

nia-

cin,

62.6

$\mathrm{mg}$;

pan-

tothe

nic

acid,

26.8

$\mathrm{mg}$;

folic

acid, 
1.14Digestible methionine (\%)0.36Calcium (\%)0.67Available phosphorus (\%)0.33 $3^{1}$ Supplied per

kilogram of diet: $\mathrm{Cu}$, 9.9 $\mathrm{mg}$; $\mathrm{Zn}$, 142.5

$\mathrm{mg}$;

$\mathrm{Fe}$, 242.5

$\mathrm{mg}$;

$\mathrm{Mn}$,

68.7

mg;

Se,

0.36

mg; I,

0.64

mg;

vit-

amin

A, 11500

IU;

vitamin

D3,

3250

IU;

vit-

amin

E, 91

mg;

vit-

amin

K3, 3

mg;

Digestible lysine (\%)1.14Digestible methio-

thi-

amin,

nine (\%)0.36Calcium (\%)0.67Available phosphorus (\%)0.33

8.6

Supplied per kilogram of

$\mathrm{mg}$; diet: $\mathrm{Cu}, 9.9 \mathrm{mg}$; Zn, 142.5

ribo-

$\mathrm{mg}$; Fe, $242.5 \mathrm{mg}$; Mn, 68.7

fla$\mathrm{mg}$; Se, $0.36 \mathrm{mg}$; I, $0.64 \mathrm{mg}$;

vin, vitamin A, $11500 \mathrm{IU}$; vita10.9 min D3, 3250 IU; vitamin $\mathrm{mg}$; $\mathrm{E}, 91 \mathrm{mg}$; vitamin $\mathrm{K} 3,3 \mathrm{mg}$; thiamin, $8.6 \mathrm{mg}$; riboflavin, pyr$10.9 \mathrm{mg}$; pyridoxine, 9.3 idoxi $\mathrm{mg}$; cobalamin, $0.2 \mathrm{mg}$; ne, niacin, $62.6 \mathrm{mg}$; panto9.3 thenic acid, $26.8 \mathrm{mg}$; folic $\mathrm{mg}$; acid, $1.9 \mathrm{mg}$. 


0.2
mg;
nia-
cin,
62.6
mg;
pan-
tothe
nic
acid,
26.8
mg;
folic
acid,
1.9
mg.

0.36Calcium

(\%)0.67Available phosphorus (\%)0.33 ${ }^{1}$

Sup-

plied

per

kilo-

gram

of

diet:

$\mathrm{Cu}$,

9.9

$\mathrm{mg}$;

Zn,

142.5

$\mathrm{mg}$;

$\mathrm{Fe}$,

242.5

$\mathrm{mg}$;

$\mathrm{Mn}$,

68.7

$\mathrm{mg}$;

Se,

0.36

$\mathrm{mg}$; I,

0.64

mg;

vit-

amin

Digestible methionine (\%)0.36Calcium A,

(\%)0.67Available phosphorus (\%)0.33 ${ }^{1}$ Supplied per kilo11500 gram of diet: $\mathrm{Cu}, 9.9 \mathrm{mg}$; IU; $\mathrm{Zn}, 142.5 \mathrm{mg}$; Fe, $242.5 \mathrm{mg}$; vit$\mathrm{Mn}, 68.7 \mathrm{mg}$; Se, $0.36 \mathrm{mg}$; I, amin $0.64 \mathrm{mg}$; vitamin A, 11500 D3, IU; vitamin D3, 3250 IU; 3250 vitamin E, $91 \mathrm{mg}$; vitamin IU; $\mathrm{K} 3,3 \mathrm{mg}$; thiamin, $8.6 \mathrm{mg}$; riboflavin, $10.9 \mathrm{mg}$; pyridoxine, $9.3 \mathrm{mg}$; cobalamin, $0.2 \mathrm{mg}$; niacin, $62.6 \mathrm{mg}$; pantothenic acid, $26.8 \mathrm{mg}$; folic acid, $1.9 \mathrm{mg}$.

vitamin E, 91 $\mathrm{mg}$; vitamin $\mathrm{K} 3,3$ $\mathrm{mg}$; 


$$
\begin{aligned}
& \text { thi- } \\
& \text { amin, } \\
& 8.6 \\
& \text { mg; } \\
& \text { ribo- } \\
& \text { fla- } \\
& \text { vin, } \\
& 10.9 \\
& \text { mg; } \\
& \text { pyr- } \\
& \text { idoxi } \\
& \text { ne, } \\
& 9.3 \\
& \text { mg; } \\
& \text { co- } \\
& \text { bal- } \\
& \text { amin, } \\
& 0.2 \\
& \text { mg; } \\
& \text { nia- } \\
& \text { cin, } \\
& 62.6 \\
& \text { mg; } \\
& \text { pan- } \\
& \text { tothe } \\
& \text { nic } \\
& \text { acid, } \\
& 26.8 \\
& \text { mg; } \\
& \text { folic } \\
& \text { acid, } \\
& 1.9 \\
& \text { mg. }
\end{aligned}
$$




3250
IU;
vit-
amin
E, 91
mg;
vit-
amin
K3, 3
mg;
thi-
amin,
8.6
mg;
ribo-
fla-
vin,
10.9
mg;
pyr-
idoxi
ne,
9.3
mg;
co-
bal-
amin,
0.2
mg;
nia-
cin,
62.6
mg;
pan-
tothe
nic
acid,
26.8
mg;
folic
acid,
1.9
mg.

$0.33^{1}$ Supplied per kilo-

Available phosphorus (\%)0.33 ${ }^{1}$ Supplied per gram of kilogram of diet: $\mathrm{Cu}, 9.9$ diet: mg; Zn, 142.5 mg; Fe, 242.5 $\mathrm{Cu}$, $\mathrm{mg}$; Mn, $68.7 \mathrm{mg}$; Se, 0.36 $\mathrm{mg}$; I, $0.64 \mathrm{mg}$; vitamin A, 11500 IU; vitamin D3, 3250 IU; vitamin E, $91 \mathrm{mg}$; vitamin $\mathrm{K} 3,3 \mathrm{mg}$; thiamin, 8.6 9.9 $\mathrm{mg}$; $\mathrm{Zn}$, 142.5 $\mathrm{mg}$; riboflavin, $10.9 \mathrm{mg}$; $\mathrm{mg}$; Fe, pyridoxine, $9.3 \mathrm{mg}$; cobalamin, $0.2 \mathrm{mg}$; niacin, $62.6 \mathrm{mg}$; pantothenic acid, 242.5

$\mathrm{mg}$;

$\mathrm{Mn}$, $26.8 \mathrm{mg}$; folic acid, $1.9 \mathrm{mg}$. 


0.36
mg; I,
0.64
mg;
vit-
amin
A,
11500
IU;
vit-
amin
D3,
3250
IU;
vit-
amin
E, 91
mg;
vit-
amin
K3, 3
mg;
thi-
amin,
8.6
mg;
ribo-
fla-
acid,
min;
acid,
10.9
mg.
mg;
pan-
pyr-
idoxi
ne,
9.3
mg;
co-
bal-
amin,
0.2
mg;
nia-
cin,
mathe
mic

${ }^{1}$ Supplied per kilogram of diet: $\mathrm{Cu}, 9.9 \mathrm{mg}$; Zn, $142.5 \mathrm{mg}$; Fe, $242.5 \mathrm{mg}$; Mn, $68.7 \mathrm{mg}$; Se, $0.36 \mathrm{mg}$; $0.64 \mathrm{mg}$; vitamin A, $11500 \mathrm{IU}$; vitamin D3, $3250 \mathrm{IU}$; vitamin E, $91 \mathrm{mg}$; vitamin K3, $3 \mathrm{mg}$; thiamin, 8.6 $\mathrm{mg}$; riboflavin, $10.9 \mathrm{mg}$; pyridoxine, $9.3 \mathrm{mg}$; cobalamin, $0.2 \mathrm{mg}$; niacin, $62.6 \mathrm{mg}$; pantothenic acid, $26.8 \mathrm{mg}$; folic acid, $1.9 \mathrm{mg}$. 
2.7. Effect of combination of Xyn, Afd and FE on growth and health of piglets fed bran-added diet 2.7.1. Animal experiment design

The experimental animal protocols for this study were approved by the Animal Care and Use Committee of South China Agricultural University. Du$\operatorname{roc} \times($ Landrace $\times$ Yorkshire) crossbred piglets at $35 \mathrm{~d}$ of age were raised individually in stainless steel cages and maintained in an environmentally controlled room $\left(25^{\circ} \mathrm{C}\right)$. After acclimation to the environment and basal diet for 1 week, a total of 270 postweaning piglets were picked out and allocated into 3 treatment groups: control group (received bran-added diet), $X$ group (received bran-added diet supplemented with $1600 \mathrm{U} / \mathrm{kg}$ $\mathrm{Xyn})$, and $(\mathrm{X}+\mathrm{A}+\mathrm{F})$ group (received bran-added diet supplemented with $1600 \mathrm{U} / \mathrm{kg} \mathrm{Xyn,}$ $0.8 \mathrm{U} / \mathrm{kg}$ Afd and $4 \mathrm{U} / \mathrm{kg}$ FE). The enzyme preparations were provided by AsiaPac Co., Ltd (Dongguan, China) and these dosages were selected based on several preliminary experiments in pigs. Each group had 6 replicates (15 piglets/replicate). The composition and nutrient levels of bran-added diet are shown in Table 1 . The experiment lasted for $21 \mathrm{~d}$. The initial body weight $(9.70 \pm 0.50 \mathrm{~kg})$ of piglets was similar across all replicates. Piglets had free access to water and feed. Feed consumption and final body weight (FBW) were recorded at $21 \mathrm{~d}$ of the experiment for calculating average daily gain (ADG), average daily feed intake (ADFI) and feed conversion ratio (FCR). Meanwhile, individual pigs were examined for diarrhea two times per day during the experimental period to calculate the diarrhea rate, which was evaluated by fecal consistency scoring using a four-grade system [29]. The occurrence of diarrhea was defined as maintaining a score of 3 for two days or a score of 4 for one day. The diarrhea rate (\%) was calculated as the sum of the number of diarrheal piglets divided by the total number of piglets.

At $21 \mathrm{~d}$ of the experiment, one piglet per replicate was randomly selected and slaughtered by severing the jugular vein, followed by separation of the intestine and collection of the digesta of cecum and colon.

2.7.2. Assay of intestinal $\mathrm{pH}$ value and volatile fatty acid (VFA) concentrations of piglets

Immediately after cutting open the cecum and colon, the $\mathrm{pH}$ value of digesta in the mid-segments of cecum and colon was measured with a DELTA320 pH meter (Mettler Toledo, Switzerland). The VFA concentrations in cecal and colonic digesta were determined using gas chromatography GC-17A (Shimadzu, Japan) with a flame ionization detector fitted with a Nukol FFAP capillary column ( $30 \mathrm{~m} \times 0.32 \mathrm{~mm} \times 0.25 \mu \mathrm{m}$, Supelco, USA) according to the method reported elsewhere [30].

\subsection{Statistical analysis}

Data were presented as mean \pm standard deviation and analyzed by one-way ANOVA using SPSS 18.0 software. Differences among treatment groups were detected by using Duncan's multiple range test. Significance was defined as $P<0.05$ and $0.05 \leq P<$ 0.10 was considered to be a tendency toward significance.

\section{Results and Discussion}

\subsection{Enzymological characteristics of Xyn, Afd and FE}

The activity of Xyn peaked at $\mathrm{pH} 5.0$ and declined gently with the deviation of $\mathrm{pH}$ value from 5.0 (Figure 1A). Xyn maintained more than $50 \%$ relative activity in a wide temperature range $\left(30^{\circ} \mathrm{C} \sim 90^{\circ} \mathrm{C}\right)$, with the value peaking at $40^{\circ} \mathrm{C}$ (Figure $\left.1 \mathrm{~B}\right)$. In comparison, Afd had the highest activity at $\mathrm{pH} 6.0$ and retained more than $60 \%$ relative activity when $\mathrm{pH}$ value ranged from 3.0 to 9.0 (Figure 1C). The relative activity of Afd was kept above $60 \%$ at the temperature range of $30^{\circ} \mathrm{C} \sim 85^{\circ} \mathrm{Cand}$ maximized at $40^{\circ} \mathrm{C}$ (Figure 1D). FE kept over $60 \%$ relative activity during $\mathrm{pH} 3.0$ to 9.0 and the optimum $\mathrm{pH}$ for its activity was 5.0 (Figure 1E). FE retained more than $60 \%$ relative activity during $30^{\circ} \mathrm{C}$ to $80^{\circ} \mathrm{C}$, with $50^{\circ} \mathrm{C}$ being the optimal temperature (Figure 1F).

Strikingly, both Xyn, Afd and FE retained more than $50 \%$ relative activity when incubated at $\mathrm{pH} 3.0$ for $30 \mathrm{~min}$ (Figure 2). Xyn and Afd maintained more than $70 \%$ relative activity while FE retained approximately $40 \%$ relative activity when subjected to high 
temperature $\left(85^{\circ} \mathrm{C}\right)$ treatment for $3 \mathrm{~min}$. The above results suggested that both $\mathrm{Xyn}$, Afd and FE particularly the first two had a relatively high thermal endurance and acid resistance, which enabled a feasibility of these enzymes applied in diet to withstand the hostile conditions in diet processing and gastric digestion. 

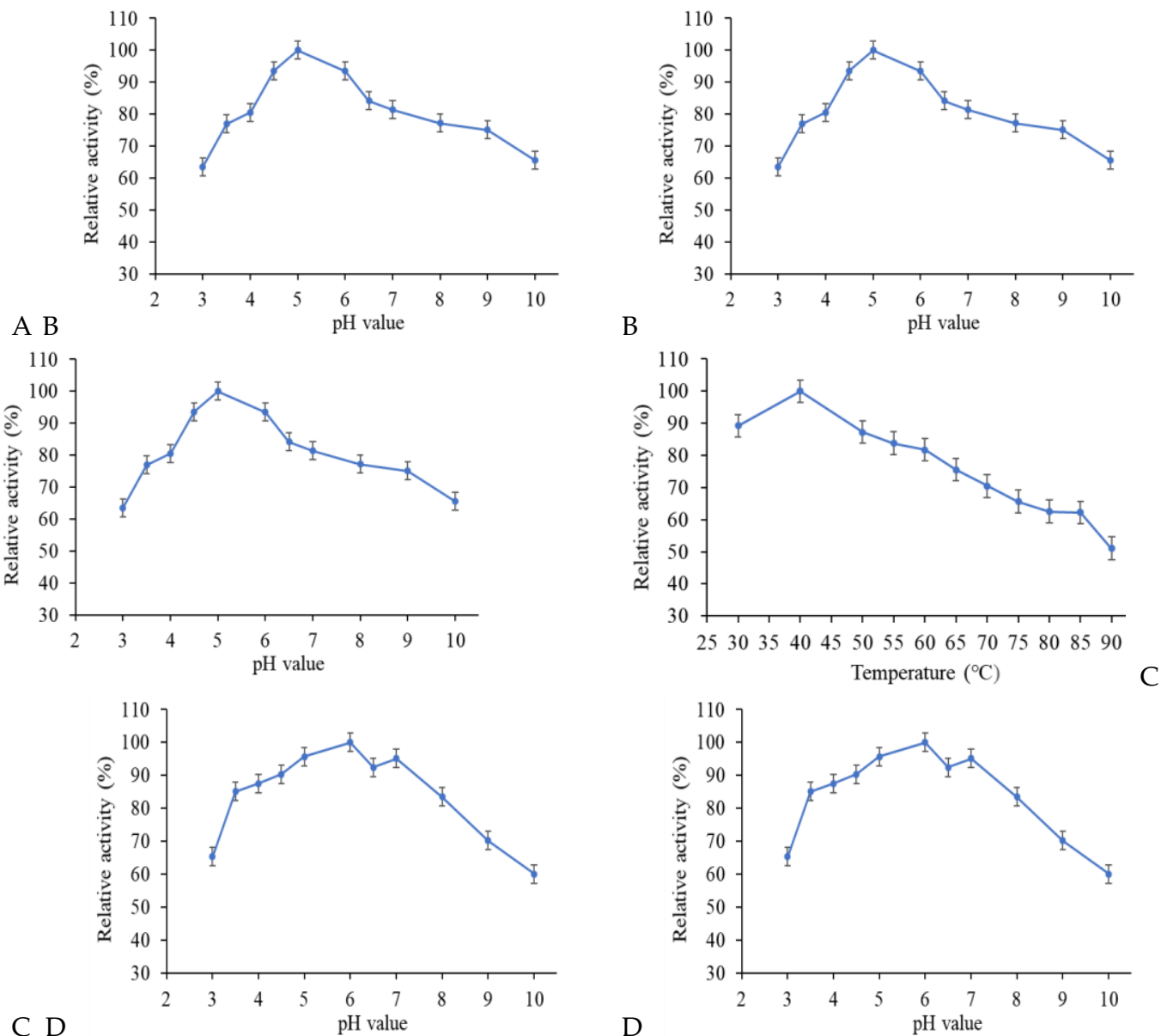

C D

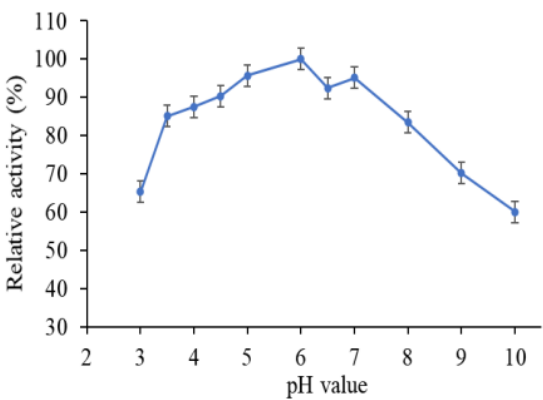

E $F$

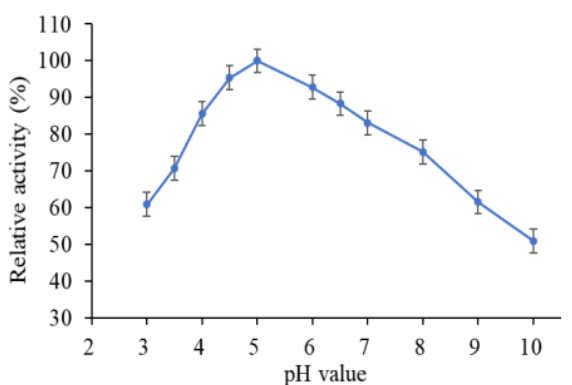

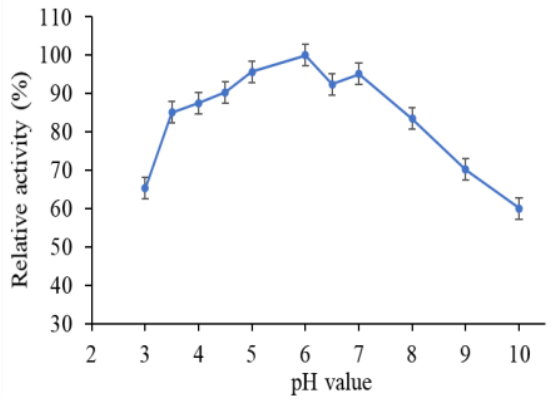
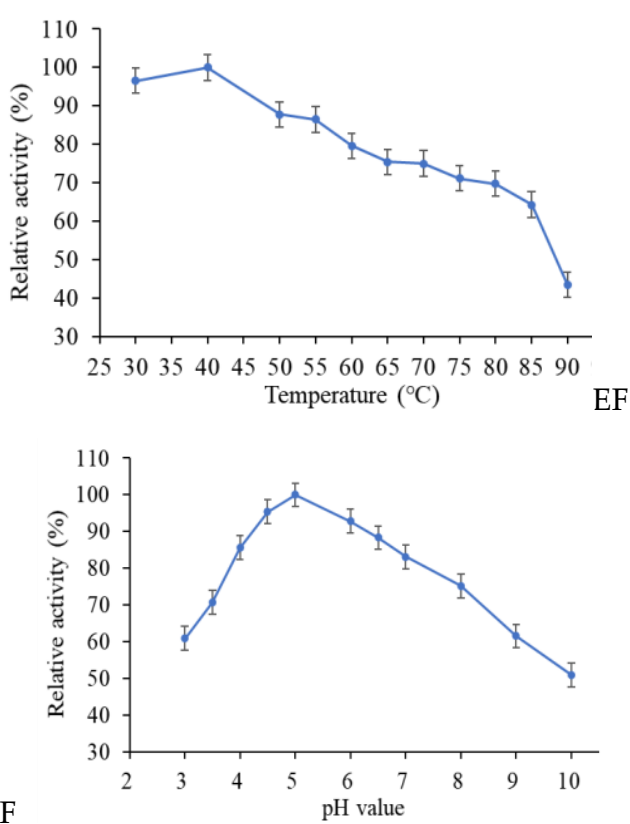

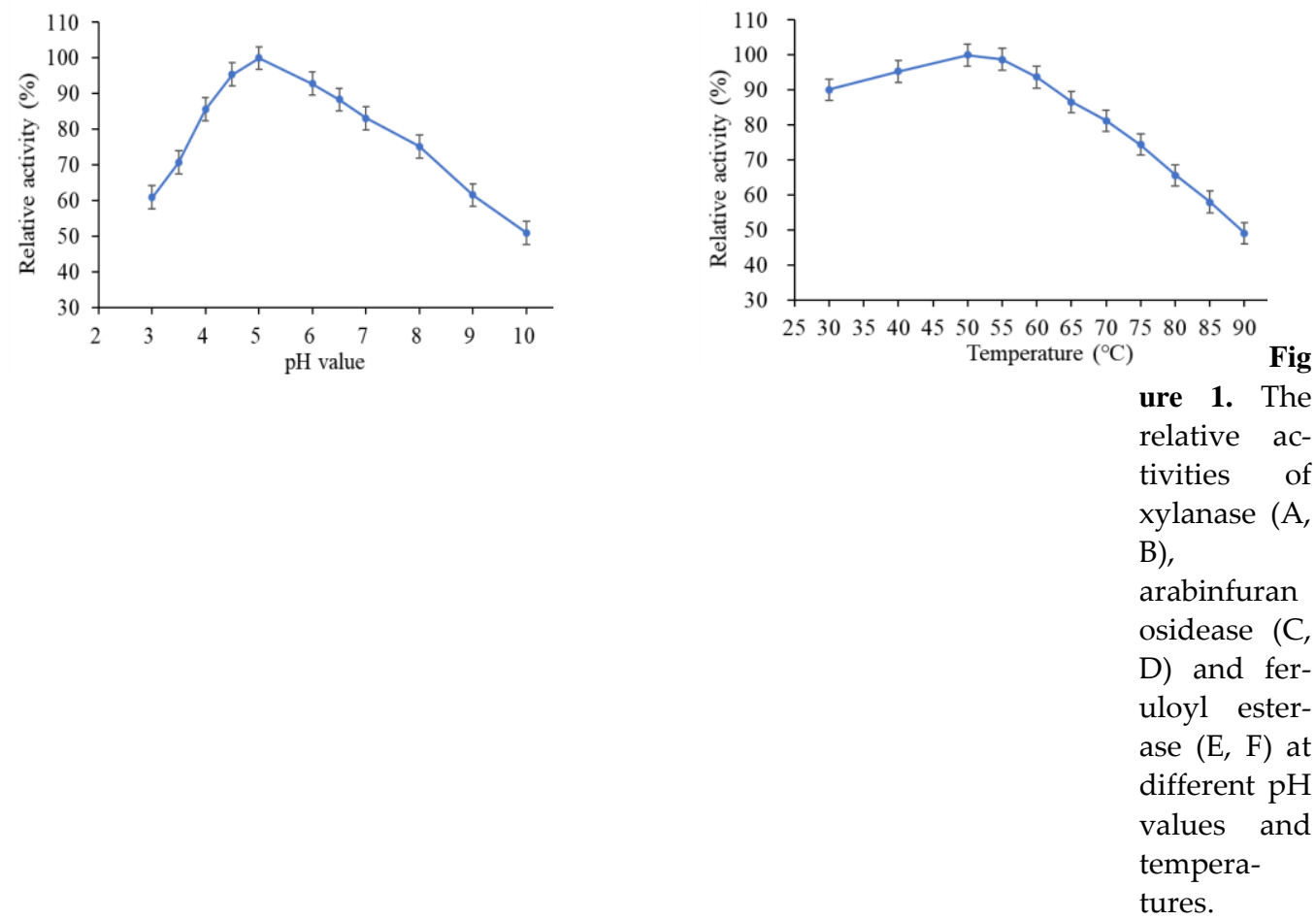

Figure 1. The relative activities of xylanase (A, B), arabinfuranosidease (C, D) and feruloyl esterase $(\mathrm{E}, \mathrm{F})$ at different $\mathrm{pH}$ values and temperatures.

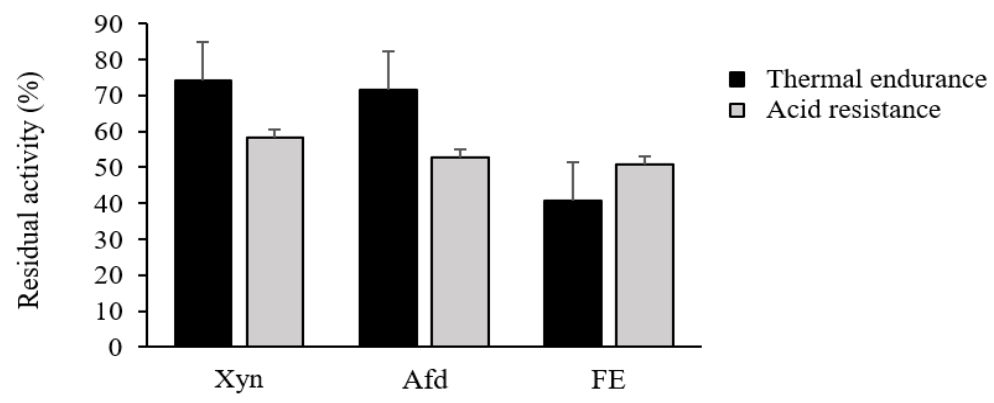

Figure 2. The thermal endurance and acid resistance of xylanase (Xyn), arabinfuranosidease (Afd) and feruloyl esterase (FE).

\subsection{Effect of Xyn combined with Afd on degradation of Abx in DSWB}

Xyn treatment alone sharply increased $(P<0.05)$ the RRS from DSWB (Figure 3), hinting that Xyn exerted a leading role among consortium of xylanolytic enzymes in degradation of $A b x$ that subsequently released reducing sugars [3]. Despite the capacity of Xyn to stochastically break Abx backbone into smaller fragments, the existence of arabinose groups attached to the backbone could somewhat reduce the degradation efficieny of Abx through hampering the recognition of cleavage sites within the backbone by Xyn $[9,10]$. Nevertheless, this steric hindrance can be alleviated by the usage of Afd that removes arabinose groups from side chains, thus providing a convenience for further action of Xyn on Abx $[3,10]$. In support of this view, we noted that combination of Xyn and Afd at a ratio ranging from 3000:1 to 100:1 resulted in a more pronounced $(P<0.05)$ RRS from DSWB relative to Xyn acting alone (Figure 3), validating a synergistic interaction between Xyn and Afd on degradation of Abx in DSWB [31,32]. Intriguingly, there was almost no elevation in the RRS with the increased ratio of Xyn dose to Afd dose. Thereby, the combination of Xyn treatment $(3 \mathrm{U} / \mathrm{g})$ and Afd treatment $(0.001 \mathrm{U} / \mathrm{g})$ at a ratio of 3000:1 was selected for further analysis. 


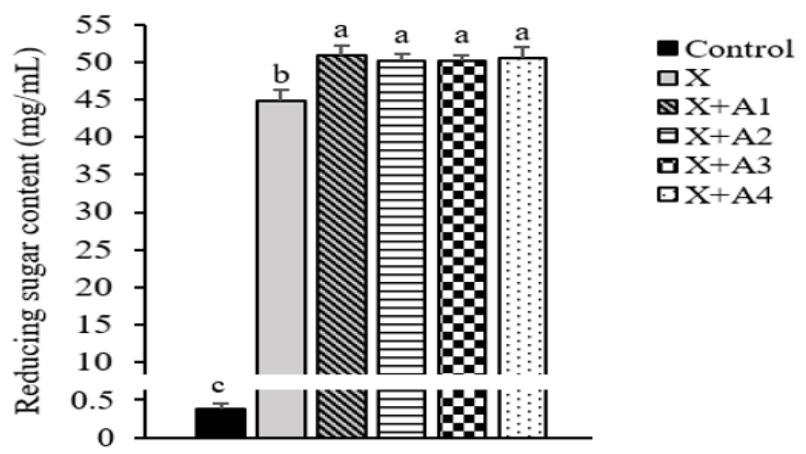

Figure 3. Effect of combination of xylanase (Xyn) and arabinfuranosidease (Afd) on the release of reducing sugar in destarched wheat bran. ${ }^{\mathrm{a}-\mathrm{c} V a l u e s}$ with different superscripts differ significantly $(P<0.05)$. Control: free of enzymes; X: $3 \mathrm{U} / \mathrm{g}$ Xyn; $(X+\mathrm{A} 1): 3 \mathrm{U} / \mathrm{g}$ Xyn $+0.001 \mathrm{U} / \mathrm{g}$ Afd; $(\mathrm{X}+\mathrm{A} 2): 3 \mathrm{U} / \mathrm{g}$ Xyn $+0.003 \mathrm{U} / \mathrm{g}$ Afd; $(X+A 3): 3 \mathrm{U} / \mathrm{g}$ Xyn $+0.006 \mathrm{U} / \mathrm{g}$ Afd; $(\mathrm{X}+\mathrm{A} 4): 3 \mathrm{U} / \mathrm{g}$ Xyn $+0.03 \mathrm{U} / \mathrm{g}$ Afd.

\subsection{Effect of Xyn combined with FE on degradation of Abx in DSWB}

As exhibited in Figure 4, combination of Xyn and FE at varying ratios (from 3000:1 to 100:1) displayed an advantage $(P<0.05)$ over $X y n$ alone in promoting the RRS from DSWB, which emphasized a synergy between Xyn and FE on degradation of Abx in DSWB. It has been indicated that the arabinose residues linked in Abx backbone can further be substituted at the O-5 position by ferulic acid ester [3], an another essential side chain group of Abx to impede the depolymerization of Xyn, therefore retarding $\mathrm{Abx}$ degradation [33]. This steric hindrance effect can be attenuated by the action of FE capable of catalyzing hydrolysis of ferulic acid ester bonds attached to Abx side chains [34], which might account for the observed advantage of combination of Xyn and FE over Xyn alone. Similarly, it was reported that the release of xylo-oligosaccharide or total reducing sugars from Abx-riched crop products under the synergy between Xyn and FE was higher than that under Xyn acting alone $[16,20]$. Notably, due to generation of the highest RRS from DSWB, the combination of Xyn (3 U/g) and FE $(0.006 \mathrm{U} / \mathrm{g})$ at a ratio of 500:1 was used for further analysis.

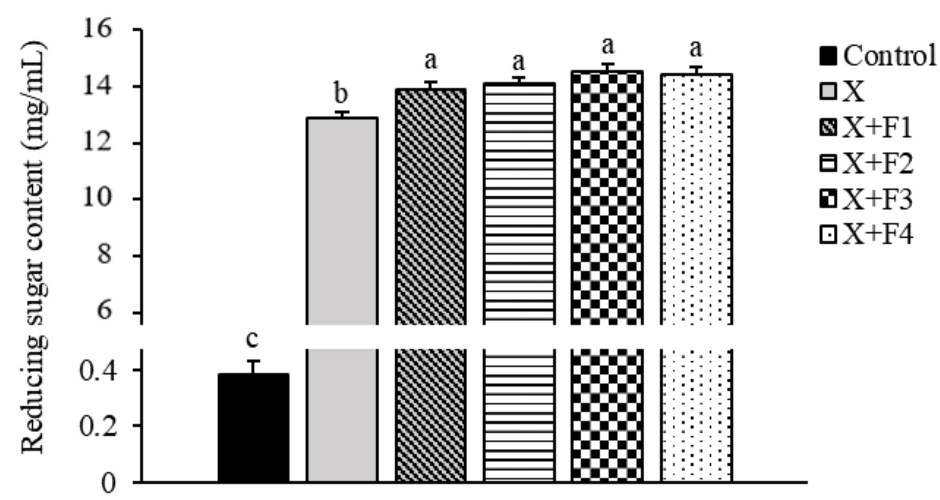

Figure 4. Effect of combination of xylanase (Xyn) and feruloyl esterase (FE) on the release of reducing sugar in destarched wheat bran. ${ }^{\mathrm{a}-\mathrm{c}}$ Different superscript letters in column indicate significant differences $(P<0.05)$. Control: free of enzymes; $X: 3 \mathrm{U} / \mathrm{g} X y n ;(X+F 1): 3 \mathrm{U} / \mathrm{g}$ Xyn $+0.001 \mathrm{U} / \mathrm{g} F E$; (X+F2): $3 \mathrm{U} / \mathrm{g}$ Xyn + $0.003 \mathrm{U} / \mathrm{g} \mathrm{FE} ;(\mathrm{X}+\mathrm{F} 3): 3 \mathrm{U} / \mathrm{g}$ Xyn + $0.006 \mathrm{U} / \mathrm{g} \mathrm{FE} ;(\mathrm{X}+\mathrm{F} 4): 3 \mathrm{U} / \mathrm{g}$ Xyn + $0.03 \mathrm{U} / \mathrm{g}$ FE. 3.4. Synergy among Xyn, Afd and FE on degradation of Abx in different brans

In the present study, WB-SF, WB-IF, OB-SF and OB-IF were employed as different Abx sources to validate the synergy among $\mathrm{Xyn}$, Afd and FE at optimum doses $(3,0.001$ and $0.006 \mathrm{U} / \mathrm{g}$, respectively). The yield of WB-SF, WB-IF, OB-SF and OB-IF was 2.75\%, $40.25 \%, 7.4 \%$ and $8.7 \%$, respectively (Table 2 ), while the extraction ratio of these bran fibers was $94.82 \%, 95.83 \%, 98.67 \%$ and $95.18 \%$, respectively.

As shown in Figure 5A, combining Xyn with Afd increased $(P<0.05)$ the RRS from both WB-SF, WB-IF, OB-SF and OB-IF as compared with Xyn acting alone, while combination of Xyn and FE caused an increase in the RRS from OB-SF and OB-IF rather than WB-SF and WB-IF. The difference between the degrading actions on OB fiber and WB 
fiber might be somewhat related to their different level of ferulic acid ester that can be targeted by FE [35]. Abx contains a linear backbone of xylopyranosyl residues that are substituted with various degrees of side chains including L-arabinose and ferulic acid groups $[3,36]$. For most cereals, arabinose monomer seems to be the main group linked as the side chain of Abx [36]. It was thus deduced that Afd might elicit a more momentous role than $\mathrm{FE}$ in removing the side chains of $\mathrm{Abx}$, probably being more beneficial for depolymerization of Abx in cereal brans by Xyn. This might account for the current result that combining Xyn with Afd led to a higher RRS from Abx sources (especially WB fiber) in comparison with combination of Xyn and FE, as well as the result that the synergy degree between Xyn and Afd was obviously greater than that between Xyn and FE (Figure 5B). Strikingly, integration of Xyn, Afd and FE induced an increase $(P<0.05)$ in the RRS from all bran fibers relative to either combination of Xyn and Afd or of Xyn and FE, highlighting that combination of Xyn, Afd and FE had a pronounced superiority over combination of Xyn and Afd or of Xyn and FE in promoting Abx degradation of different brans, which corresponded to the higher synergy degree among these three enzymes than that between Xyn and Afd or between Xyn and FE (Figure 5B). The above results raised a necessity of combining Xyn with Afd and FE to promote full degradation of Abx in brans, most likely due to that cooperation of debranching enzymes (Afd and FE) was more efficient in eliminating the spatial obstacles (branch points) that limited the formation of enzyme-substrate complex in the backbone of Abx [37], thus favoring the access of Xyn to Abx and its depolymerization.

Table 2. Extraction of fibers from wheat bran and oat bran

\begin{tabular}{cccccc}
\hline & \multicolumn{2}{c}{ Wheat bran } & & \multicolumn{2}{c}{ Oat bran } \\
\cline { 2 - 3 } & Yield (\%) & Extraction ratio (\%) & & Yield (\%) & Extraction ratio (\%) \\
\hline Soluble fiber & 2.75 & 94.82 & & 7.40 & 98.67 \\
Insoluble fiber & 40.25 & 95.83 & & 8.70 & 95.18 \\
\hline
\end{tabular}


A.

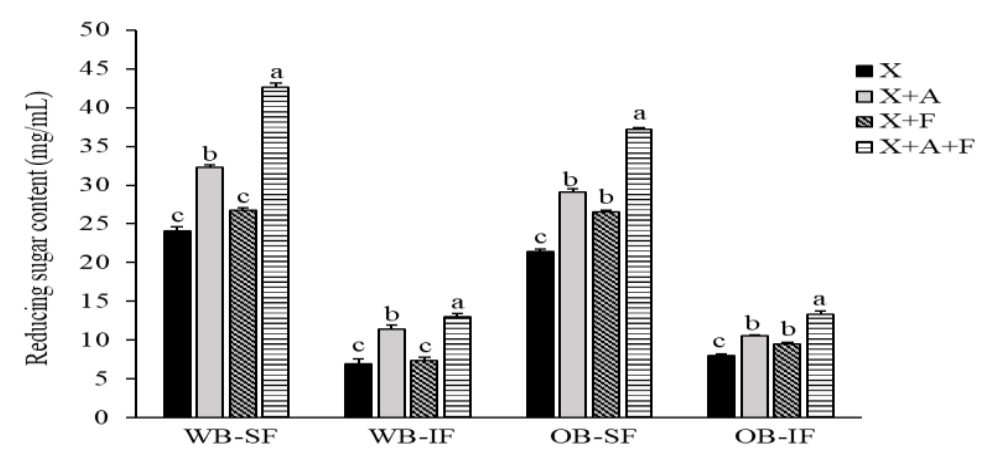

B.

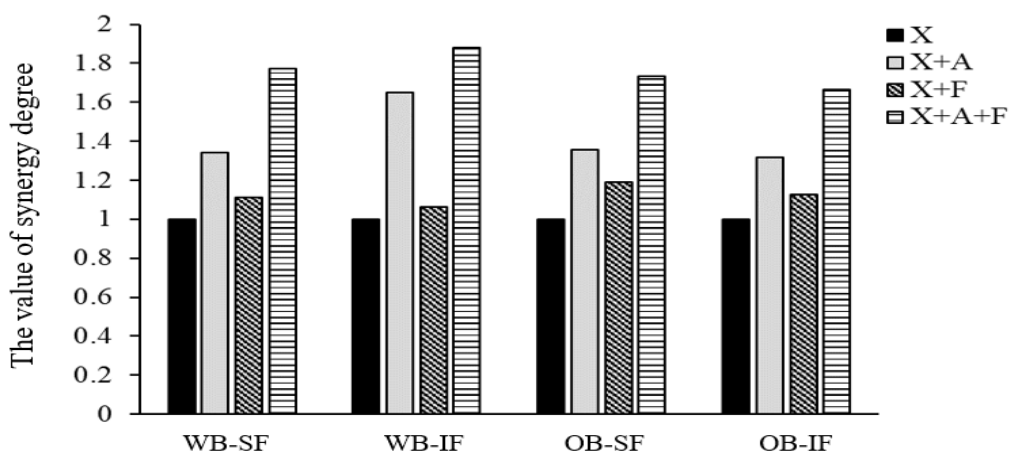

Figure 5. Synergistic action among xylanase (Xyn), arabinfuranosidease (Afd) and feruloyl esterase (FE). (A) the release of reducing sugar from different arabinoxylan sources; (B) the value of synergy degree among Xyn, Afd and FE. a-cValues with different superscripts differ significantly $(P<0.05)$. WB, wheat bran; WB-SF, soluble fiber of wheat bran; WB-IF, insoluble fiber of wheat bran; OB, oat bran; WB-SF, soluble fiber of oat bran; WB-IF, insoluble fiber of oat bran. X: $3 \mathrm{U} / \mathrm{g}$ Xyn; $(X+A): 3 \mathrm{U} / \mathrm{g}$ Xyn + $0.001 \mathrm{U} / \mathrm{g}$ Afd; $(\mathrm{X}+\mathrm{F}): 3 \mathrm{U} / \mathrm{g}$ Xyn $+0.006 \mathrm{U} / \mathrm{g} \mathrm{FE} ;(\mathrm{X}+\mathrm{A}+\mathrm{F}): 3 \mathrm{U} / \mathrm{g}$ Xyn $+0.001 \mathrm{U} / \mathrm{g}$ Afd +0.006 $\mathrm{U} / \mathrm{g}$ FE.

\subsection{Effect of combination of Xyn, Afd and FE on in vitro digestion of bran-added diet}

Xyn treatment was legitimately effective in promoting digestion of dietary fibers in animals [38-40], nevertheless, little study was available concerning the effect of Xyn combined with debranching enzymes on nutrient digestibility of piglets. In this study, we found that treatment with combination of Xyn, Afd and FE had a potential to improve nutrient digestibility, as manifested by the increasing trends $(P<0.10)$ of in vitro digestibility of dry matter, crude protein, crude ash and gross energy of bran-added diet (Figure 6). It was possible that the observed superiority of combination of Xyn, Afd and FE over Xyn alone in Abx degradation might translate into a benefit for cell wall destruction of brans or some other plant feedstuffs in diet, which potentially allowed an enhanced access of digestive enzymes to the cell wall and/or intracellular constituents of these ingredients $[15,41]$, consequently favoring digestion of dietary nutrients. Alternatively, the mechanism underlying the increased nutrient digestibility might be associated with that the efficient degradation of Abx in diet under the synergy among Xyn, Afd and FE profited the lowering of digesta viscosity, which could facilitate a sufficient contact between digesta and digestive enzymes with a subsequent improvement of nutrient digestion $[4,5]$. 


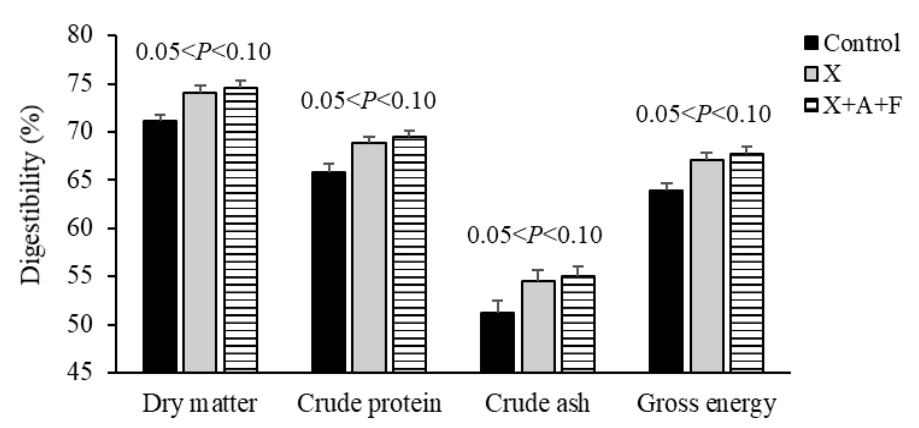

Figure 6. Effect of combination of xylanase (Xyn), arabinfuranosidease (Afd) and feruloyl esterase (FE) on in vitro nutrient digestibility of bran-added diet. Control: without enzymes; X: 3 U/g Xyn; $(\mathrm{X}+\mathrm{A}+\mathrm{F}): 3 \mathrm{U} / \mathrm{g}$ Xyn $+0.001 \mathrm{U} / \mathrm{g}$ Afd $+0.006 \mathrm{U} / \mathrm{g}$ FE.

3.6. Effect of combination of Xyn, Afd and FE on growth performance of piglets fed bran-added diet Combination of Xyn, Afd and FE did not affect ADFI of piglets, but had an advantage over Xyn alone to induce increasing trends $(P<0.10)$ of FBW and ADG along with a decreasing trend $(P<0.10)$ of FCR of postweaning piglets fed bran-added diet (Figures 7A D). Although numerous studies evidenced a variable efficacy of Xyn treatment alone in promotion of growth performance of pigs [38-40], there was little study regarding the effect of combination of $\mathrm{Xyn}, \mathrm{Afd}$ and $\mathrm{FE}$ on pig growth performance. The results obtained herein revealed a potential of consortium of Xyn, Afd and FE to improve growth rate and feed efficiency of piglets, which was most likely responsible by the observed elevation in nutrient digestibility of diet treated with combination of Xyn, Afd and FE. With respect to the diarrhea rate of piglets, it was found to be reduced $(P<0.05)$ by the treatment with either Xyn alone or combination of Xyn, Afd and FE (Figure 7E), with a lower $(P<0.05)$ diarrhea rate in combined treatment group versus $X y n$ alone group. It was probable that the elevated nutrient digestibility of diet induced by these enzyme consortium led to reductions of nutrient residuals utilized by certain pathogens in hindgut, presumptively decreasing the prevalence of diarrhea of piglets [42,43]. Alternatively, the efficient degradation of Abx in diet under the synergy among Xyn, Afd and FE could lower the viscosity of chyme, which was hypothesized to avoid overgrowth of intestinal harmful bacteria and in turn reduce diarrhea rate of piglets [4, 44].

A.

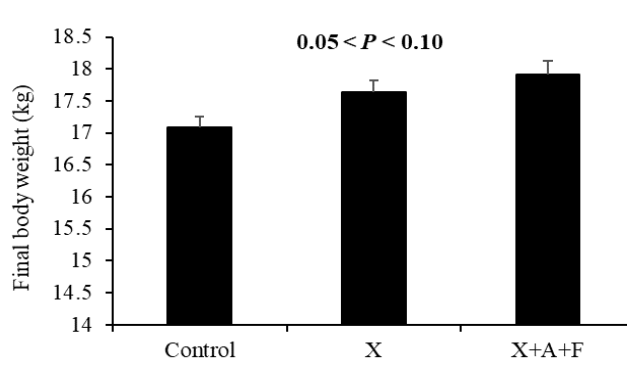

C.

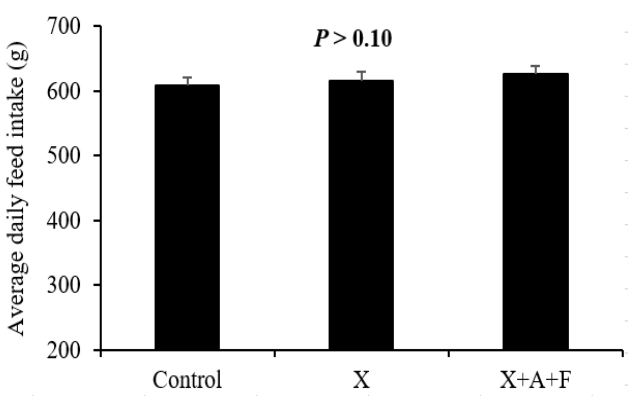

E.
B.

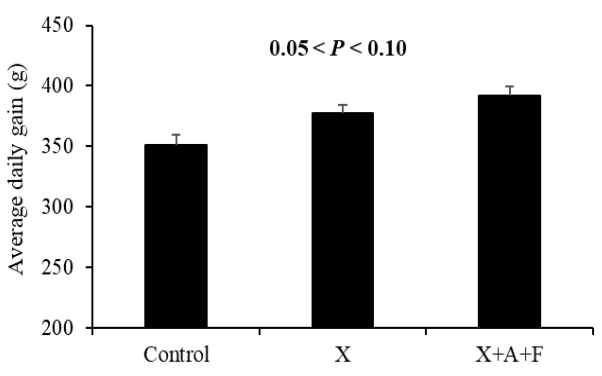

D.

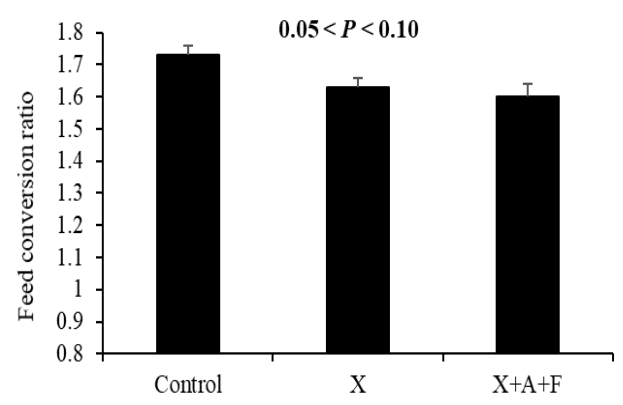




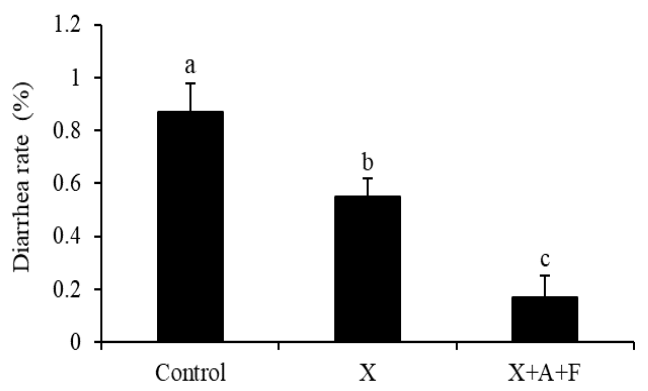

Figure 7. Effect of combination of xylanase (Xyn), arabinfuranosidease (Afd) and feruloyl esterase (FE) on growth performance of piglets received bran-added diet. (A) final body weight; (B) average daily gain; (C) average daily feed intake; (D) feed conversion ratio; (E) diarrhea rate. ${ }^{\mathrm{a}-\mathrm{C}}$ Values with different superscripts differ significantly $(P<0.05)$. Control: treatment without enzymes; $\mathrm{X}$ : treatment with $1600 \mathrm{U} / \mathrm{kg}$ Xyn; $(\mathrm{X}+\mathrm{A}+\mathrm{F})$ : treatment with combination of $1600 \mathrm{U} / \mathrm{kg}$ Xyn, $0.8 \mathrm{U} / \mathrm{kg}$ Afd and $4 \mathrm{U} / \mathrm{kg}$ FE.

\subsection{Effects of combination of Xyn, Afd and FE on intestinal VFA profile and $p H$ value of piglets fed bran-added diet}

Treatment with combination of Xyn, Afd and FE or Xyn alone increased $(P<0.05)$ the concentrations of acetic acid and total VFA in cecum and colon as well as butyric acid in cecum of piglets fed bran-added diet, with higher $(P<0.05)$ concentrations of these parameters in combined treatment group versus Xyn treatment alone (Figures $8 \mathrm{~A} \sim \mathrm{D}$ ). Moreover, there was an elevation $(P<0.05)$ in cecal and colonic propionic acid concentration in response to the treatment with combination of Xyn, Afd and FE (Figure 8B). VFA produced by bacterial fermentation of dietary fibers have long been characterized as a typical improver of gut health of piglets [30]. It was reported that dietary Xyn treatment could modulate fermentation pattern of gut microbiota and increase the concentrations of certain VFA in gut of pigs fed Abx-riched diet [38,39]. However, the role of synergy among Xyn, Afd and FE on gut health of pigs remains unknown. Our results revealed a distinct advantage of combination of Xyn, Afd and FE over Xyn alone in improving intestinal VFA profile, which might be associated with that the degradation of dietary Abx into certain oligoscaccharides under the synergy among Xyn, Afd and FE improved gut fermentation by several beneficial bacteria (namely the prebiotic effect) [4,39].

The elevated concentrations of certain VFA in gut induced by combination of Xyn, Afd and FE were postulated to translate into a lower $\mathrm{pH}$ value of gut. Although treatment with Xyn alone had little influence $(P>0.05)$ on intestinal $\mathrm{pH}$ (Figure $8 \mathrm{E})$, we indeed found a reduction $(P<0.05)$ of cecal $\mathrm{pH}$ value together with a decreasing trend $(P<$ 0.10 ) of colonic $\mathrm{pH}$ value in piglets treated with combination of Xyn, Afd and FE when compared with either control or Xyn alone, which roughly coincided to the simultaneous increases in cecal and colonic concentrations of VFA as a result of treatment with combination of Xyn, Afd and FE. We speculated that the increased concentrations of VFA along with the decrease of $\mathrm{pH}$ value in gut due to treatment with combination of $\mathrm{Xyn}$, Afd and FE were at least partially responsible for the observed reduction of diarrhea rate of piglets, because acid microenvironment in gut could resist pathogen invasion as well as boost gut health and growth performance of piglets [45,46]. 
A.

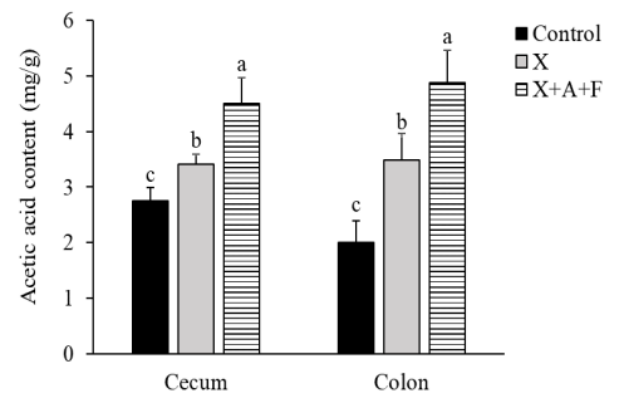

C.

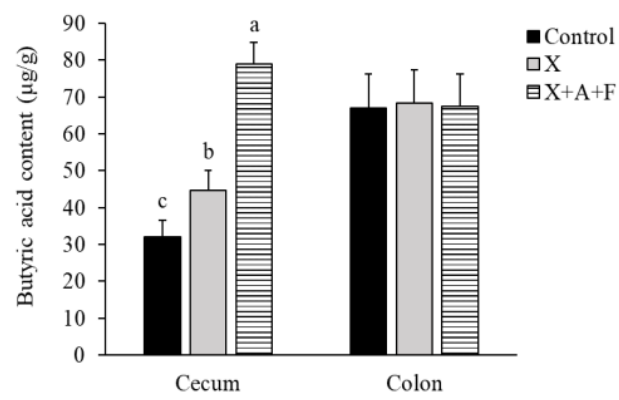

E.

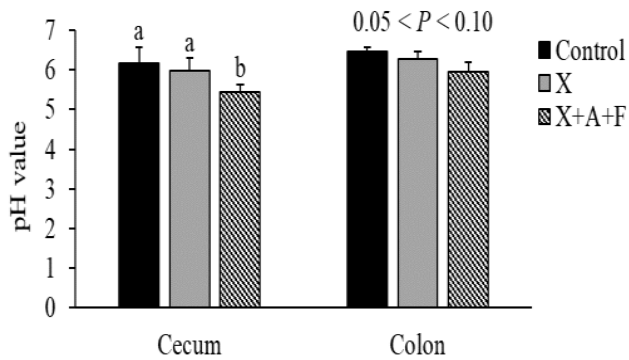

Figure 8. Ef-

fects

of

combination

of Figure 8 .

Effects of

combination

of xylanase

(Xyn),

arabinfurano

sidease (Afd)

and feruloyl

esterase (FE)

on volatile

fatty acid

concentra-

tions and $\mathrm{pH}$

value in in-

testinal di-

gesta of pig-

lets received

bran-added

diet. ${ }^{a-c}$ Values

with differ-
B.

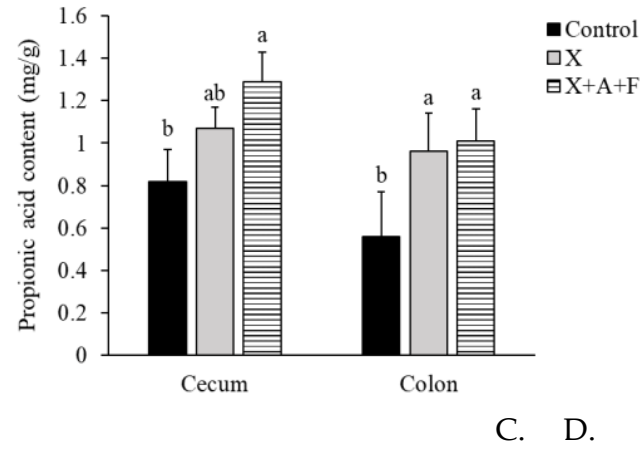

D.

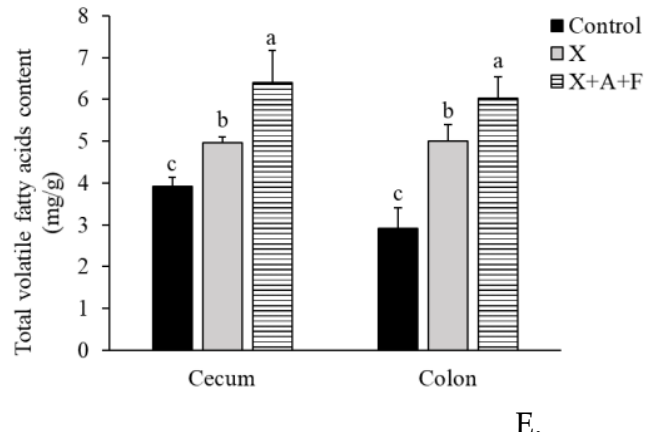

Figure 8.

Effects of combination of Figure 8. Effects of combination of xylanase (Xyn), arabinfuran osidease

(Afd) and feruloyl esterase (FE) on volatile fatty acid concentra-

tions and $\mathrm{pH}$ value in intestinal

digesta of

piglets received

bran-added

diet.

a-cValues

with different superscripts differ significantly $(P<0.05)$. Control: treatment 
ent super-

scripts differ

significantly

$(P<0.05)$.

Control:

treatment

without en-

zymes; $\mathrm{X}$ :

treatment

with 1600

$\mathrm{U} / \mathrm{kg}$ Xyn;

$(\mathrm{X}+\mathrm{A}+\mathrm{F})$ :

treatment

with combi-

nation of

$1600 \mathrm{U} / \mathrm{kg}$

Xyn, $0.8 \mathrm{U} / \mathrm{kg}$

Afd and 4

U/kg FE. without en-

zymes; $\mathrm{X}$ : treatment

with 1600

$\mathrm{U} / \mathrm{kg}$ Xyn;

$(\mathrm{X}+\mathrm{A}+\mathrm{F})$ :

treatment

with com-

bination of

$1600 \mathrm{U} / \mathrm{kg}$

Xyn, $\quad 0.8$

$\mathrm{U} / \mathrm{kg}$ Afd

and $4 \mathrm{U} / \mathrm{kg}$

FE.

nation of Figure 8. Effects of combination of xylanase (Xyn), arabinfuranosidease (Afd) and feruloyl esterase (FE) on volatile fatty acid concentrations and $\mathrm{pH}$ value in intestinal digesta of piglets

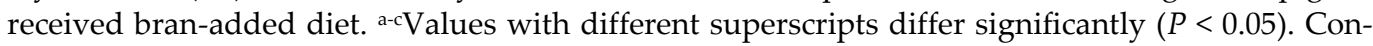
trol: treatment without enzymes; $X$ : treatment with $1600 \mathrm{U} / \mathrm{kg} \mathrm{Xyn} ;(\mathrm{X}+\mathrm{A}+\mathrm{F})$ : treatment with combination of $1600 \mathrm{U} / \mathrm{kg}$ Xyn, $0.8 \mathrm{U} / \mathrm{kg}$ Afd and $4 \mathrm{U} / \mathrm{kg}$ FE.

\section{Conclusions}

The present study evidenced relatively high thermal endurance and acid resistance of Xyn, Afd and FE, which were suitable to be applied in animal diet. Combining these enzymes had a superiority over Xyn acting alone and its combination with Afd or FE in degradation of Abx in different bran fibers. Dietary treatment with combination of Xyn, Afd and FE had advantages over Xyn alone to improve nutrient digestion, growth performance and intestinal VFA profile of piglets received bran-added diet.

Author Contributions: Conceptualization, W.W.W.; methodology, Z.Z.Z.; software, Q.Y.C. and H.Y.; validation, W.W.W. and Z.Z.Z.; formal analysis, C.M.Z. and Z.M.D.; investigation, Z.Z.Z.; resources, D.Y.F. and J.J.Z.; data curation, J.J.Z.; writing-original draft preparation, W.W.W.; writing-review and editing, D.Y.F. and J.J.Z.; visualization, J.J.Z.; supervision, D.Y.F. and J.J.Z.; project administration, D.Y.F. and J.J.Z.; funding acquisition, J.J.Z. All authors have read and agreed to the published version of the manuscript.

Funding: This research was funded by the National Natural Science Foundation (No. 31872390) and the Modern Feed Industry Innovation Team Project of Guangdong Province (No. 2019KJ115) as well as the Public Welfare Research and Capacity Building Project of Guangdong Province (No. 2016A020210104).

Institutional Review Board Statement: This study was conducted in accordance with the protocols of the Animal Care and Use Committee of South China Agricultural University (Approval no. SCAU20200416).

Data Availability Statement: The data presented in this study are available from the corresponding author on reasonable request..

Acknowledgments: The authors thank Yiliang Chen, Yuanbin Liu and Shujie Liang (South China Agricultural University, Guangzhou, China) for their assistance in sample collection.

Conflicts of Interest: The authors declare no conflict of interest.

\section{References}

1. Knudsen, K.E.B. Fiber and nonstarch polysaccharide content and variation in common crops used in broiler diets. Poult. Sci. 2014, 93, 2380-2393.

2. Naidu, D.S.; Hlangothi, S.P.; John, M.J. Bio-based products from xylans: A review. Carbohyd. Polym. $2018,179,28-41$. 
3. Malgas, S.; Mafa, M.S.; Mkabayi, L.; Pletschke, B.I. A mini review of xylanolytic enzymes with regards to their synergistic interactions during hetero-xylan degradation. World J. Microb. Biot. 2019, 35, 187.

4. Baker, J.T.; Duarte, M.E.; Holanda, D.M.; Kim, S.W. Friend or Foe? Impacts of dietary xylans, xylooligosaccharides, and xylanases on intestinal health and growth performance of monogastric animals. Animals 2021, 11, 609.

5. Sun, H.; Cozannet, P.; Ma, R.; Zhang, L.; Huang, Y.K.; Preynat, A.; Sun, L.H. Effect of concentration of arabinoxylans and a carbohydrase mixture on energy, amino acids and nutrients total tract and ileal digestibility in wheat and wheat by-product-based diet for pigs. Anim. Feed Sci. Technol. 2020, 262, 11480.

6. Fu, H.K.; Mo, W.X.; Shen, X.N.; Li, B. Impact of centrifugation treatment on enzymatic hydrolysis of cellulose and xylans in poplar fibers with high lignin content. Bioresour. Technol. 2020, 316, 123866.

7. Xu, D.M.; Ding, Z.T.; Bai, J.; Ke, W.C.; Zhang, Y.X.; Li, F.H.; Guo, X.S. Evaluation of the effect of feruloyl esterase-producing Lactobacillus plantarum and cellulase pretreatments on lignocellulosic degradation and cellulose conversion of co-ensiled corn stalk and potato pulp. Bioresour. Technol. 2020, 310, 123476.

8. Moreira, L.R.S.; Filho, E.X.F. Insights into the mechanism of enzymatic hydrolysis of xylans. Appl. Microbiol. Biotechnol. 2016, $100,5205-5214$.

9. Kouzounis, D.; Hageman, J.A.; Soares, N.; Michiels, J.; Schols, H.A. Impact of xylanase and glucanase on oligosaccharide formation, carbohydrate ermentation patterns, and nutrient utilization in the gastrointestinal tract of broilers. Animals 2021, 11, 1285.

10. Raweesri, P.; Riangrungrojana, P.; Pinphanichakarn, P. alpha-LArabinofuranosidase from Streptomyces sp PC22: Purification, characterization and its synergistic action with xylanolytic enzymes in the degradation of xylans and agricultural residues. Bioresour. Technol. 2008, 99, 8981-8986.

11. Liu, S.J.; Soomro, L.; Wei, X.; Yuan, X.F.; Gu, T.Y.; Li, Z.; Wang, Y.L.; Bao, Y.M.; Wang, F.Z.; Wen, B.T. Directed evolution of feruloyl esterase from Lactobacillus acidophilus and its application for ferulic acid production. Bioresour. Technol. 2021, 332, 124967.

12. Nieter, A.; Kelle, S.; Linke, D.; Berger, R.G. Feruloyl esterases from Schizophyllum commune to treat food industry side-streams. Bioresour. Technol. 2016, 220, 38-46.

13. Rahmani, N.; Kahar, P.; Lisdiyanti, P.; Hermiati, E., Lee, J., Yopi, Prasetya, B.; Ogino, C.; Kondo, A. Xylanase and feruloyl esterase from actinomycetes cultures could enhance sugarcane bagasse hydrolysis in the production of fermentable sugars. $\mathrm{Bi}$ osci. Biotechnol. Biochem. 2018, 82, 904-915.

14. de Oliveira, D.M.; Finger-Teixeira, A.; Mota, T.R.; Salvador, V.H.; Moreira-Vilar, F.C.; Molinari, H.B.; Mitchell, R.A.; Marchiosi, R.; Ferrarese-Filho, O.; dos Santos, W.D. Ferulic acid: a key component in grass lignocellulose recalcitrance to hydrolysis. Plant Biotechnol. J. 2015, 13, 1224-1232.

15. Dyk, J.S.V.; Pletschke, B.I. A review of lignocellulose bioconversion using enzymatic hydrolysis and synergistic cooperation between enzymes-factors affecting enzymes, conversion and synergy. Biotechnol. Adv. 2012, 30, 1458-1480.

16. Wu, H.L.; Li, H.L.; Xue, Y.; Luo, G.; Gan, L.H.; Liu, J.; Mao, L.H.; Long, M.N. High efficiency co-production of ferulic acid and xylooligosaccharides from wheat bran by recombinant xylanase and feruloyl esterase. Biochem. Eng. J. 2017, 120, 41-48.

17. Saleh, A.A.; Kirrella, A.A.; Abdo, S.E.; Mousa, M.M.; Badwi, N.A.; Ebeid, T.A.; Nada A.L.; Mohamed, M.A. Effects of dietary xylanase and arabinofuranosidase combination on the growth performance, lipid peroxidation, blood constituents, and immune response of broilers fed low-energy diets. Animals 2019, 9, 467.

18. Ravn, J.L.; Glitso, V.; Pettersson, D.; Ducatelle, R.; Van-Immerseel, F.; Pedersen, N.R. Combined endo-beta-1,4-xylanase and alpha-L-arabinofuranosidase increases butyrate concentration during broiler cecal fermentation of maize glucurono-arabinoxylan. Anim. Feed Sci. Technol. 2018, 236, 159-169.

19. Cozannet, P.; Kidd, M.T.; Neto, R.M.; Geraert, P.A. Next-generation non-starch polysaccharide-degrading, multi-carbohydrase complex rich in xylanase and arabinofuranosidase to enhance broiler feed digestibility. Poult. Sci. 2017, 96, $2743-2750$.

20. Mukherjee, G.; Singh, R.K.; Mitra, A.; Sen, S.K. Ferulic acid esterase production by Streptomyces sp. Bioresour. Technol. 2007, 98, 211-213.

21. Wang, R.N.; Yang, J.S.; Jang, J.M.; Liu, J.W.; Zhang, Y.; Liu, L.; Yuan, H.L. Efficient ferulic acid and xylo-oligosaccharides production by a novel multi-modular bifunctional xylanase/feruloyl esterase using agricultural residues as substrates. Bioresour. Technol. 2020, 297, 122487.

22. Terrasan, C.R.F.; Temer, B.; Duarte, M.C.T.; Carmona, E.C. Production of xylanolytic enzymes by Penicillium janczewskii. Bioresour. Technol. 2010, 101, 4139-4143.

23. Kanaya, K.; Tada, S.; Mori, B.; Takahashi, R.; Ikegami, S.; Kurasawa, S.; Okuzaki, M.; Mori, Y.; Innami, S. A simplified modification of the AOAC official method for determination of total dietary fiber using newly developed enzymes: Preliminary interlaboratory study. J. AOAC Int. 2007, 90, 225-237.

24. Fahey, G.J.; Novotny, L.; Layton, B.; Mertens, D.R. Critical factors in determining fiber content of feeds and foods and their ingredients. J. AOAC Int. 2019, 102, 52-62.

25. Kumar, R.; Wyman, C.E. Effects of cellulase and xylanase enzymes on the deconstruction of solids from pretreatment of poplar by leading technologies. Biotechnol. Prog. 2009, 25, 302-314.

26. Chen, Y.X.; Shen, D.; Zhang, L.L.; Zhong, R.Q.; Liu, Z.Q.; Liu, L.; Chen, L.; Zhang, H.F. Supplementation of non-starch polysaccharide enzymes cocktail in a corn-miscellaneous meal diet improves nutrient digestibility and reduces carbon dioxide emissions in finishing pigs. Animals 2020, 10, 232. 
27. Zhu, X.F.; Wang, L.Y.; Zhang, Z.; Ding, L.R.; Hang, S.Q. Combination of fiber-degrading enzymatic hydrolysis and lactobacilli fermentation enhances utilization of fiber and protein in rapeseed meal as revealed in simulated pig digestion and fermentation in vitro. Anim. Feed Sci. Technol. 2021, 278, 115001.

28. National Research Council (NRC) 2012. Nutrient requirements of swine, 11th revised edition. National Academies Press, Washington, DC, USA.

29. Barnett, K.L.; Kornegay, E.T.; Risley, C.R.; Lindemann, M.D.; Schurig, G.G. Characterization of creep feed consumption and its subsequent effects on immune response, scouring index and performance of weanling pigs. J. Anim. Sci. 1989, 67, 2698-2708.

30. Li, Q.Y.; Peng, X.Y.; Burrough, E.R.; Sahin, O.; Gould, S.A.; Gabler, N.K.; Loving, C.L.; Dorman, K.S.; Patience, J.F. Dietary soluble and insoluble fiber with or without enzymes altered the intestinal microbiota in weaned pigs challenged with enterotoxigenic E. coli F18. Front. Microbiol. 2020, 11, 1110.

31. Vangsoe, C.T.; Bonnin, E.; Joseph-Aime, M.; Saulnier, L.; Neugnot-Roux, V.; Knudsen, K.E.B. Improving the digestibility of cereal fractions of wheat, maize, and rice by a carbohydrase complex rich in xylanases and arabinofuranosidases: an in vitro digestion study. J. Sci. Food Agr. 2021, 101, 1910-1919.

32. Goncalves, T.A.; Damasio, A.R.L.; Segato, F.; Alvarez, T.M.; Bragatto, J.; Brenelli, L.B.; Citadini, A.P.S.; Murakami, M.T.; Ruller, R.; Paes-Leme, A.F.P.; Prade, R.A.; Squina, F.M. Functional characterization and synergic action of fungal xylanase and arabinofuranosidase for production of xylooligosaccharides. Bioresour. Technol. 2012, 119, 293-299.

33. Akin, D.E. Plant cell wall aromatics: influence on degradation of biomass. Biofuels. Bioprod. Biorefin. $2008,2,288-303$.

34. Nghiem, N.P.; Montanti, J.; Johnston, D.B.; Drapcho, C. Fractionation of corn fiber treated by soaking in aqueous ammonia (SAA) for isolation of hemicellulose B and production of C5 sugars by enzyme hydrolysis. Appl. Biochem. Biotechnol. 2011, 164, 1390-1404.

35. Lavinia, F.C.; Dan, C.V. Thermal processing for the release of phenolic compounds from wheat and oat bran. Biomolecules 2019, 10, 21.

36. Naidu, D.S.; Hlangothi, S.P.; John, M.J. Bio-based products from xylans: A review. Carbohyd. Polym. $2018,179,28-41$.

37. Valenzuela, S.V.; Diaz, P.; Pastor, F.I. Xyn11E from Paenibacillus barcinonensis BP-23: a LppX-chaperone-dependent xylanase with potential for upgrading paper pulps. Appl. Microbiol. Biotechnol. 2014, 98, 5949-5957.

38. Mejicanos, G.A.; Gonzalez-Ortiz, G.; Nyachoti, C.M. Effect of dietary supplementation of xylanase in a wheat-based diet containing canola meal on growth performance, nutrient digestibility, organ weight, and short-chain fatty acid concentration in digesta when fed to weaned pigs. J. Anim. Sci. 2020, 98, skaa064.

39. Petry, A.L.; Patience, J.F. Xylanase supplementation in corn-based swine diets: a review with emphasis on potential mechanisms of action. J. Anim. Sci. 2020, 98, skaa318.

40. Lu, H.; Yan, H.; O'Neill, H.M.M.; Bradley, C.; Bedford, M.R.; Wilcock, P.; Nakatsu, C.H.; Adeola, O.; Ajuwon, K.M. Effect of timing of postweaning xylanase supplementation on growth performance, nutrient digestibility, and fecal microbial composition in weanling pigs. Can. J. Anim. Sci. 2020, 100, 27-36.

41. Oliveira, D.M.; Mota, T.R.; Oliva, B.; Segato, F.; Marchiosi, R.; Ferrarese, O.; Faulds, C.B.; dos Santos, W.D. Feruloyl esterases: Biocatalysts to overcome biomass recalcitrance and for the production of bioactive compounds. Bioresour. Technol. 2019, 278, 408-423.

42. Rhouma, M.; Fairbrother, J.M.; Beaudry, F.; Letellier, A. Post weaning diarrhea in pigs: risk factors and non-colistin-based control strategies. Acta Vet. Scand. 2017, 59, 31.

43. Zhang, Z.Y.; Li, Z.Q.; Zhao, H.; Chen, X.L.; Tian, G.; Liu, G.M.; Cai, J.Y.; Jia, G. Effects of drinking water temperature and flow rate during cold season on growth performance, nutrient digestibility and cecum microflora of weaned piglets. Animals 2020, $10,1048$.

44. Gao, J.; Yin, J.; Xu, K.; Li, T.J.; Yin, Y.L. What is the impact of diet on nutritional diarrhea associated with gut microbiota in weaning piglets: a system review. Biomed Res. Int. 2019, 2019, 6916189.

45. Tugnoli, B.; Giovagnoni, G.; Piva, A.; Grilli, E. From acidifiers to intestinal health enhancers: how organic acids can improve growth efficiency of pigs. Animals 2020, 10, 134.

46. Rodrigues, L.M.; Neto, T.O.D.; Garbossa, C.A.P.; Martins, C.C.D.; Garcez, D.; Alves, L.K.S.; de-Abreu, M.L.T.; Ferreira, R.A.; Cantarelli, V.D. Benzoic acid combined with essential oils can be an alternative to the use of antibiotic growth promoters for piglets challenged with E. coli F4. Animals 2020, 10, 1978. 